\title{
Water entry of spinning spheres
}

\author{
TADD T. TRUSCOTT AND ALEXANDRA H. TECHET $\dagger$
}

Department of Mechanical Engineering, Massachusetts Institute of Technology, Cambridge, MA 02139, USA

(Received 24 August 2007 and in revised form 3 December 2008)

The complex hydrodynamics of water entry by a spinning sphere are investigated experimentally for low Froude numbers. Standard billiard balls are shot down at the free surface with controlled spin around one horizontal axis. High-speed digital video sequences reveal unique hydrodynamic phenomena which vary with spin rate and impact velocity. As anticipated, the spinning motion induces a lift force on the sphere and thus causes significant curvature in the trajectory of the object along its descent, similar to a curveball pitch in baseball. However, the splash and cavity dynamics are highly altered for the spinning case compared to impact of a sphere without spin. As spin rate increases, the splash curtain and cavity form and collapse asymmetrically with a persistent wedge of fluid emerging across the centre of the cavity. The wedge is formed as the sphere drags fluid along the surface, due to the no-slip condition; the wedge crosses the cavity in the same time it takes the sphere to rotate one-half a revolution. The spin rate relaxation time plateaus to a constant for tangential velocities above half the translational velocity of the sphere. Non-dimensional time to pinch off scales with Froude number as does the depth of pinch-off; however, a clear mass ratio dependence is noted in the depth to pinch off data. A force model is used to evaluate the lift and drag forces on the sphere after impact; resulting forces follow similar trends to those found for spinning spheres in oncoming flow, but are altered as a result of the subsurface air cavity. Images of the cavity and splash evolution, as well as force data, are presented for a range of spin rates and impact speeds; the influence of sphere density and diameter are also considered.

\section{Introduction}

The water-entry problem, by itself, is directly relevant to many different applications: from ballistics (May 1975) and ship slamming (Faltinsen \& Zhao 1997) to skipping stones (Rosellini et al. 2005) and Basilisk lizards (Glasheen \& McMahon 1996). One of the geometrically most simple objects that can be studied is the sphere. This canonical shape impacting on the free surface does not, however, yield simple hydrodynamic results, and the results are even more complex when spin is introduced (Truscott \& Techet 2006). An experimental study of the impact of a sphere, spinning transverse to its velocity, on a water surface is presented herein, offering a first look into how spin can affect water-entry behaviour.

Figure 1 shows a comparison of the non-spinning $(a)$ and spinning $(b)$ impact of a standard billiard ball on the free surface. The air cavity and splash formed by the spinning sphere vary distinctly from the axisymmetric cavity formed with no spin. The subsurface air cavity bends along the trajectory of the spinning sphere, and the splash 
(a)

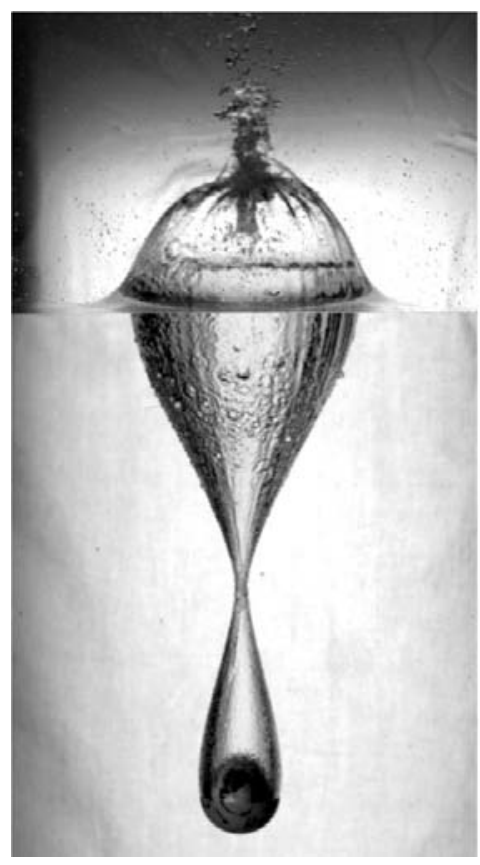

(b)

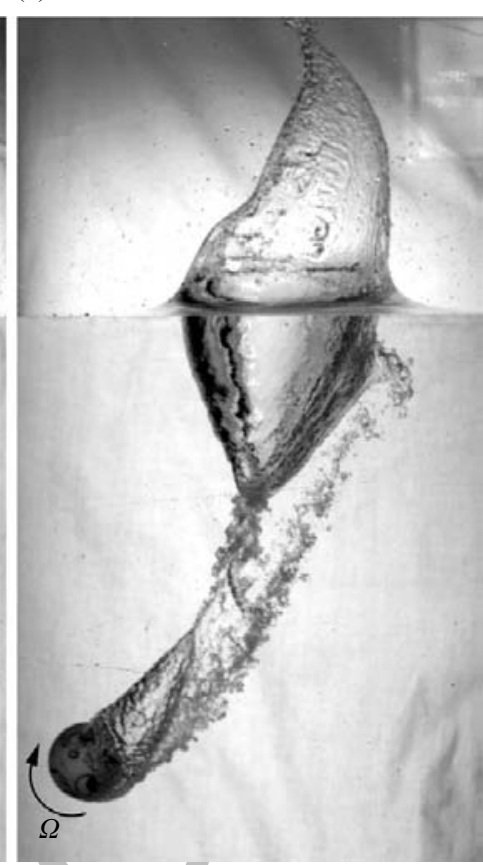

FIGURE 1. Images of the cavity and splash formations by $(a)$ non-spinning and $(b)$ spinning sphere $(d=0.057 \mathrm{~m})$ after impact into water. Impact speeds are $V(a)=5.95 \mathrm{~m} \mathrm{~s}^{-1}(F r=7.9)$ and $V(b)=5.45 \mathrm{~m} \mathrm{~s}^{-1}(F r=7.3)$. Case $(b)$ has a spin rate at impact of $251 \mathrm{rad} \mathrm{s}^{-1}$, in the clockwise direction. Both images are taken at the same time after impact $(\mathrm{t}=102 \mathrm{~ms})$.

curtain grows vertically and collapses asymmetrically. For the spinning water-entry problem, valuable insight into the physics can be drawn from both water entry and spinning sphere research.

\subsection{Water-entry problem}

Numerous experiments on water entry of bodies seek to qualitatively and quantitatively characterize the hydrodynamic phenomena generated by and forces acting on the impacting object. Among the first to study such phenomena, von Karman (1929) investigated the forces exerted on a sea plane float during landing. Subsequent early impact studies typically focus on ballistics investigations in military laboratories. The experiments of May (1975) are some of the most extensive studies of free surface impact for naval ordinance applications. His research focuses on the formation of the air cavity in the wake of spherical projectiles with high-impact velocities.

High-speed imaging techniques are critical for capturing the rapidly evolving stages of impact hydrodynamics and have been used since early water-entry tests. Worthington \& Cole (1897) present some of the earliest images of the splash cavity created by falling objects, which were captured using single spark photography. Today, digital high-speed cameras are widely used for imaging water-entry hydrodynamics. Extensive experimental investigations of water entry by spheres and projectiles are presented in Bell (1924), May \& Woodhull (1948, 1950), Richardson (1948), May (1951), May \& Hoover (1963) and Abelson (1970). 
Investigations by May (1952) of the vertical entry of missiles (steel spheres) into water indicate that Froude scaling is a good first approximation to use when describing cavity behaviours such as deep closure. Several other researchers assert, however, that the instantaneous Reynolds number is a better description of the cavity hydrodynamics than the impact Froude number (e.g. Gilbarg \& Anderson 1948).

The effect of before- and after-body shape on the impact is also of interest to researchers in the area of naval ballistics. May \& Woodhull (1948) conclude that the cavity shape is not dependent on the nose shape of the projectile for a given drag force. Shi, Itoh \& Takami (2000) image bullets shot vertically into a tank at $342 \mathrm{~m} \mathrm{~s}^{-1}$, qualitatively considering the bullets' supercavitating behaviour; they find that for blunt leading edge projectiles, the after-body shape can significantly affect the splash formation.

Atmospheric pressure can also be considered a factor affecting the impact problem. Gilbarg \& Anderson (1948), at the Naval Ordinance Laboratory, investigate the dependence of the cavity formation on atmospheric pressure. They conclude that surface closure, defined when the splash crown domes over and closes, is the most important event in the development of the water cavity and greatly influences later cavity growth, and note that time to surface closure is inversely proportional to pressure. Deep closure, when the cavity pinches off below the free surface, is a function of surface closure only for early closure times up to $\sim 70 \mathrm{~m} \mathrm{~s}^{-1}$ after impact (Gilbarg \& Anderson 1948).

Even biologists are fascinated by the problem of impact. A biological air-water impact study, modelled after the Basilisk lizard, is performed by Glasheen \& McMahon (1996). Disks, representing the lizard's feet, are shot into water at low speeds (compared to most ballistics studies); high-speed video and load cells measure the forces during impact. Their results also indicate that the time between impact and cavity closure could be modelled by a single value of dimensionless time. Surface closure and cavity pinch-off have also been numerically investigated in detail for circular disks impacting the surface by Gaudet (1998) and Bergmann et al. (2006).

Lee, Longoria \& Wilson (1997) employ a two-dimensional model, based on experimental observations, for cavity formation and collapse taking into account the energy transfer between projectile and cavity wall. Their work considers relatively low impact velocities, where both a surface closure and later a deep closure, or pinch-off, occur. Data indicate that the time to deep closure, after surface closure, is approximately constant and not a function of the impact speed for any given sphere diameter. The location of deep closure, however, seems to have only a weak dependence on impact velocity, and thus Froude number was not a good scaling parameter for the range of impact speeds that they investigated (Lee et al. 1997); the velocities and Froude numbers in Lee et al. (1997) were considerably higher than those considered in the present study. Gilbarg \& Anderson (1948) also report, for low Froude numbers, that surface closure dominates cavity formation, and note that Froude number is not a useful parameter in characterizing cavity growth and collapse.

In general, research has shown that there are a few key mechanisms driving surface closure, the most important of which are Bernoulli pressure and surface tension (Birkhoff \& Isaacs 1951; Lee et al. 1997). As the cavity grows, air flows in through the splash curtain, and for low-speed impact, the local under-pressure inside the cavity is approximately $1 / 2 \rho_{\text {air }} V_{o}^{2}$. After the splash curtain domes over and closes (i.e. surface closure), the cavity continues to expand due to inertial effects of the ball moving through the fluid. Assuming the process is isentropic (Lee et al. 1997), the pressure inside the cavity decreases. Eventually, deep closure (i.e. pinch-off) occurs when the 
cavity stops expanding radially and the hydrostatic pressure of the surrounding fluid is greater than the internal cavity pressure (Gilbarg \& Anderson 1948; May 1952; Lee et al. 1997).

For impact cases where the impact velocity is sufficiently high, a small axisymmetric horizontal jet is ejected at great velocity radially outwards from the point of contact between the sphere and the liquid surface. The jet emanates horizontally at first and can travel radially outwards at speeds up to 30 times the impact velocity. The initial jet forms between 10 and $100 \mu$ s after impact, for water solutions of up to $90 \%$ glycerin (Thoroddsen et al. 2004). Thoroddsen et al. (2004) also report that surface tension and compressibility appear to have little effect on this initial jet formation. The introduction of spin also causes a similar horizontal jet to form, albeit asymmetrically. The effect of spin on the initial stages of impact will be discussed in subsequent sections of this paper.

Yarin (2006) reviews experimental and theoretical work on droplet impact onto thin liquid layers and dry surfaces, focusing on the splash crown formation and initial jetting. He notes that jetting and crowning originate from the same point irrespective of surface shape, as a result of a sharp kinematic discontinuity. Typically the velocity of the initial horizontal jet is significantly higher than the impact velocity and the formation time is very small. These results are verified by Thoroddsen (2002) for liquid drops and are similar for solid spheres impacting on the surface (Thoroddsen et al. 2004).

Moghisi \& Squire (1981) measure the forces during impact using a load cell up to a depth of one-eighth of the sphere diameter. They report that for impact velocities between 1 and $3 \mathrm{~m} \mathrm{~s}^{-1}, 1<V_{o}<3 \mathrm{~m} \mathrm{~s}^{-1}$, a maximum force occurs very quickly after impact, at times as short as $1.5 \mathrm{~m} \mathrm{~s}^{-1}$ or between one-tenth and one-fifth of the radius below the surface. Moghisi \& Squire (1980) conclude that the dependence of drag coefficient on Reynolds numbers, in the range $0.05<R e<5 \times 10^{3}$, resembles that of a sphere in a homogeneous fluid. Their work, like much of the existing theoretical work done to determine the force at impact, only considers impact up to a maximum penetration depth of half a sphere diameter. May \& Woodhull $(1948,1950)$ note that the drag coefficient declines gradually towards a value between 0.25 and 0.3 when cavity is formed; the precise shape of the curve appears to depends on the specific gravity of the impacting object.

Direct force measurements are not easily obtained for water-entry experiments. Richardson (1948) and May \& Woodhull (1948, 1950) derive force components using force balance equations and position-time curves, taken from high-speed video after the sphere is fully submerged. Kornhauser (1964) offers a review of these force models derived from experimental data. A similar force balance model for determining forces is developed in this study on spinning spheres, and data is presented in $\S 3$.

\subsection{Spinning sphere problem}

Spin, by itself, imposed on a sphere in flow, acts to induce lift and alter the flight path of an unconstrained sphere. Newton (1671) first remarks on the distinct change in the flight path of spinning tennis balls, noting their tendency towards the side that is moving the fastest. Later, Robbins (1742), interested in this problem from his experimental observations of ballistics, shows that a spinning sphere suspended as a pendulum experiences a lateral aerodynamic force. A similar force is seen for spinning cylinders; this force due to rotation is widely credited as the 'Magnus Effect' (Barkla \& Auchterlonie 1971). 
The subject of spinning spheres is of special interest to many sports fans, especially baseball, cricket and golf enthusiasts. Baseball pitchers can break their curveballs at just the right time, or throw a knuckleball, without spin, to drop at the last minute over the plate. Golfers hoping to gain loft, or fade or hook their shots, or avoid hooking their shots, rely on small spinning balls riddled with tiny dimples. A comprehensive review of sports ball aerodynamics is presented in Mehta (1985).

Attempts to predict the behaviour of spinning sports balls drive experimentalists to perform lift and drag tests, as well as flow visualization experiments on spinning spheres. Much of the experimental force data for spinning spheres is compiled in Alaways \& Hubbard (2001). Researchers studying cricket and baseball report that, for pre-critical Reynolds numbers, asymmetric boundary layer separation, due to tripping by the seams on the balls, results in increased lateral forces (Mehta 1985). Spin-induced effects also lead to asymmetrical boundary-layer transition flow on one side of the sphere, which causes large lateral forces, for example in baseball curveballs (Mehta 1985; Alaways \& Hubbard 2001) and golf-ball flight (Bearman \& Harvey 1976).

Interestingly, for subcritical Reynolds numbers, experimental measurements of the lateral forces on spinning smooth spheres in flow, by Maccoll (1928) and Davies (1949), show that the lift coefficient $C_{L}$ can be negative for small values of nondimensional spin parameter, $S=r \Omega / V$, where $r$ is the radius of the sphere, $\Omega$ is the spin rate in radians per second, and $V$ is the flight velocity. Maccoll (1928) shows that above spin parameter values of $S=0.35-0.50$, for subcritical flow around smooth spheres, the coefficient of lift becomes positive and increases steadily up to $S=1.0$, above which the lift coefficient appears to level off. The negative lift coefficients for low spin numbers $(S<0.4)$ have never been seen for roughened or dimpled spheres or those with seams (e.g. Bearman \& Harvey 1976; Watts \& Ferrer 1987; Smits \& Smith 1994; Alaways \& Hubbard 2001).

Davies (1949) presents a plausible explanation for the negative lift force as a result of an asymmetric transition from laminar to turbulent flow. As the velocity of the incoming flow approaches the limit of transition away from laminar flow, only slight perturbations are necessary to trip the flow to turbulent. Since the sphere is spinning, one side of the sphere experiences a higher relative velocity than the other, and could trip before the side with a lower relative velocity thus inducing lift in the opposite direction than anticipated. This would only be plausible for low spin parameters, above which the force of lift from circulation is greater than the imbalance due to asymmetric transition. Davies (1949) acknowledges that this explanation works only for very specific critical parameters and any changes in turbulence levels or velocity could reverse the effect. He cautions that further measurements of pressure or flows around spinning spheres are needed in this negative lift regime.

Results from Bearman \& Harvey (1976), for a dimpled sphere, show that the lift coefficient is proportional to the spin parameter $S$ and thus the lift force is proportional to $\Omega r$, the tangential velocity. Smits \& Smith (1994) measure the forces on golf balls (dimpled spheres), along with the spin decay rate, in a wind tunnel. Their results suggest that the lift coefficient has some Reynolds number $(R e)$ dependence only up to Reynolds number 100000 . Above this value there appears to be little, if any, dependence of the lift coefficient on Reynolds number. This lack of dependence at $R e>100000$ is reinforced by data in Alaways \& Hubbard (2001), who present an extensive compilation of published data for spinning spheres.

In the current investigation, spheres with nominal roughness heights of $k /(2 r)=1.4 \times 10^{-5}$ are considered. While not the main focus, nor presented herein, 


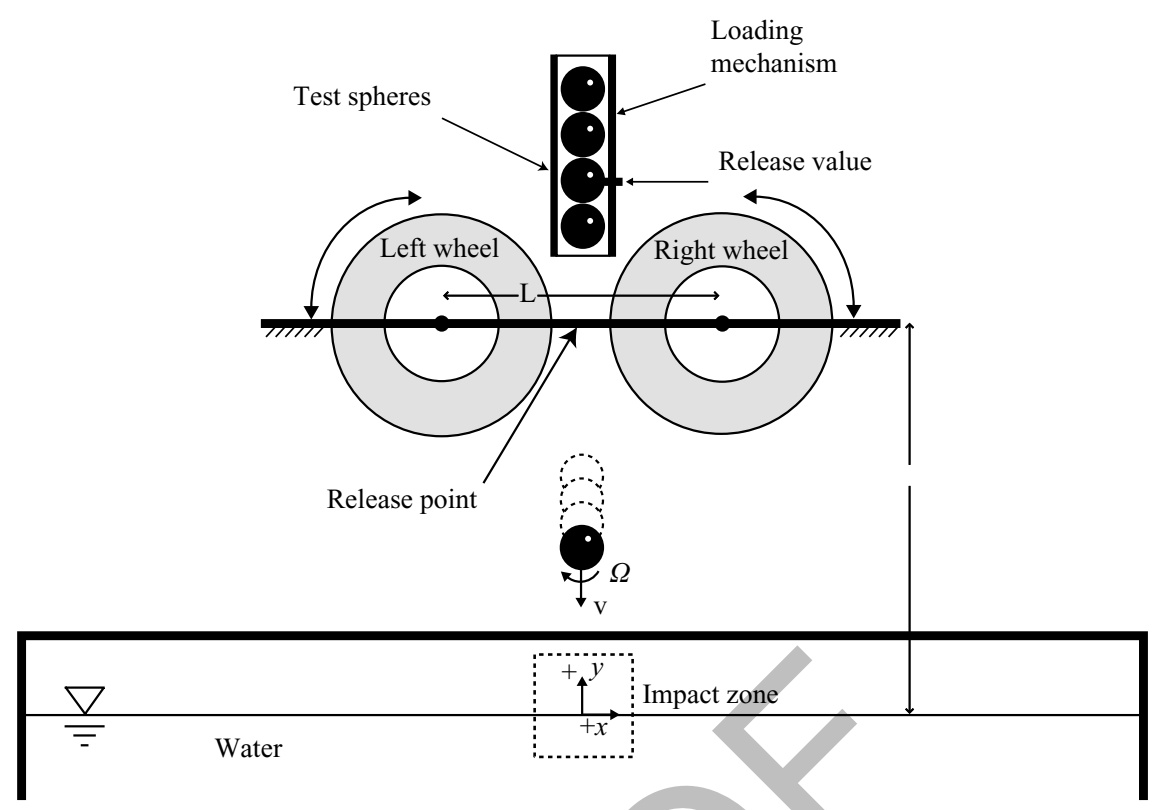

FIGURE 2. Schematic illustration of the experimental shooting apparatus. Spheres are released from the loading mechanism, dropping between the two spinning wheels, which shoot the spheres into the tank below. The speed of each wheel was independently controlled in order to change the impact and spinning speed of the projectiles. (Figure is not to scale.)

results from our own preliminary investigations in a water tunnel show good agreement with Maccoll (1928) data for the lift and drag spinning smooth spheres above spin parameter $S \approx 0.35$. The forces determined using the sphere trajectories are also similar to wind and water tunnel experiments. The effect of spin rate on the physics of the cavity dynamics, splash formation and collapse and the forces acting on the sphere are addressed in the following sections.

\section{Experimental details}

Experiments on water entry of spinning spheres were performed using standard billiard balls (diameter $d=5.72 \mathrm{~cm}$; mass $m=0.17 \mathrm{~kg}$ ) shot vertically into a tank of quiescent water. The steel-reinforced clear acrylic tank was $1.5 \mathrm{~m}$ long, $0.9 \mathrm{~m}$ wide and $1.5 \mathrm{~m}$ deep. A shooting apparatus, modelled after a baseball-style pitching machine with two rotating wheels (diameter $d_{w}=0.46 \mathrm{~m}$ ), was mounted above the tank. The spheres were released out of the loading tube and dropped between the two wheels, which fired the spheres into the water (figure 2). The ball exited the launch mechanism, with initial downwards launch velocity and spin, at a height of $1.40 \mathrm{~m}$ above the surface of the water; this maximum distance above the free surface was constrained only by the laboratory ceiling height. The wheels of the shooting mechanism imparted spin to the sphere by rotating at different speeds. Both initial impact velocity $V_{o}$ and initial spin rate $\Omega_{o}$ were varied by altering the spinning rate of wheels of the shooting mechanism. The wheel speed and ball release timing were controlled using a personal computer and a National Instruments LabView interface, which also controlled the video recording system to ensure accurate synchronized timing. 
High-speed video images of the sphere's trajectory were used to calculate the instantaneous velocity of the sphere in the $x$ - and $y$-directions. Two high-speed video systems were used: camera \#1 for the top view was an IDT X-StreamVision XS-3 camera and camera \#2 for the side view was a Redlake Motion Pro X3. It was not physically possible to place a camera directly above the impact zone as the shooting mechanism obstructed the field of view. Thus, camera \#1 was positioned adjacent to the wheels looking down at a slight oblique angle to the free surface. Camera \#2 was positioned such that it captured the motion of the sphere at least of one diameter above the water, as well as along its descent through the water, allowing the velocity just before and after impact to be determined.

Both cameras had a maximum resolution of $1280(\mathrm{~V}) \times 1024(\mathrm{H})$ pixels, however, camera \#1 had a maximum frame rate of 625 frames per second (fps) at maximum resolution, whereas camera \#2 could reach $1000 \mathrm{fps}$ at maximum resolution. When the resolution of camera \#1 was reduced in one direction, the frame rate could be increased, and thus both cameras could record at $1000 \mathrm{fps}$. At $1000 \mathrm{fps}$, camera \#1 recorded images at $480(\mathrm{H}) \times 1108(\mathrm{~V})$ pixels and camera \#2 recorded at full-pixel resolution; the exposure time was $234 \mu \mathrm{s}$ per frame for both cameras. A $28 \mathrm{~mm}$, $f / 2.8$ lens with a fully open aperture was used and a fixed field of view (FOV) was maintained on both cameras for all experiments. The cameras were synched in time with the ball release mechanism through the LabView interface.

Sufficient lighting is crucial for quality high-speed video, thus lights were placed both in front of and behind the tank. For back-lighting, 36 standard $32 \mathrm{~W}$ fluorescent bulbs were aligned vertically in a large bank that was positioned directly behind the impact zone, outside of the tank, and projected light directly towards the cameras. The bank of bare bulbs was $0.5 \mathrm{~m}$ wide $\times 1.6 \mathrm{~m}$ high. Nine separate out-of-phase ballasting units minimized the $60 \mathrm{~Hz}$ flickering effect generated by the fluorescent lights. A white sheet placed between the light bank and the impact zone diffused the back light and created a more uniform backdrop. The front illumination used six $400 \mathrm{~W}$ halogen lights positioned outside the tank and focused towards the impact region. These six lights were used to illuminate the front side of the cavity. Three of the halogen lights were mounted vertically near the water surface in an arc around the region of interest, which focused the light towards the impact zone. Three more lights, also outside the tank, were stationed approximately $1 \mathrm{~m}$ below the surface level, and focused towards the lower-half of the sphere's trajectory, again in an arc around the impact zone. The lights in front of the tank were positioned around the camera such that there was minimal glare off the tank wall and the sphere trajectory was sufficiently lit for the image processing algorithm to detect the sphere consistently.

The impact velocity and spin rate were calculated by analysing the high-speed video data. Impact speeds and initial non-zero spin rates ranged from $\left|V_{o}\right|=1.9$ to $9.9 \mathrm{~m} \mathrm{~s}^{-1}$ and $\left|\Omega_{o}\right|=13-394 \mathrm{rad} \mathrm{s}^{-1}$, respectively. For comparison, several test cases had zero spin; these cases were performed at impact velocities of $\left|V_{o}\right|=1.9-7.5 \mathrm{~m} \mathrm{~s}^{-1}$. The horizontal velocity at impact was less than $2-4 \%$ of the vertical velocity for both spinning and non-spinning cases.

The effect of spin is considered in terms of the non-dimensional ratio between tangential velocity $\Omega r$, where $r=d / 2$, and the magnitude of the velocity of the sphere $V$. The spin parameter, defined following the nomenclature of Maccoll (1928) as $S=\Omega r / V$ is akin to a Strouhal number and used in this paper to ensure consistency with previously reported data in the field of spinning spheres. Using the shooting mechanism described herein, it was possible to obtain initial impact spin parameters $S_{o}=\Omega_{o} r / V_{o}$, between 0 and 2.25 , using the spin rate and velocity at impact $\Omega_{o}$ and 
$V_{o}$. Data for cases with $S_{o}<1.4$ are presented herein. After impact, spin parameter also changed, in time, as a result of the changing instantaneous velocity and spin rate, such that $S(t)=\Omega(t) r / V(t)$.

Froude number $F r=V_{o} / \sqrt{g d}$ was used to categorize the initial impact velocity of the ball, and is only given in terms of initial impact velocity. Froude numbers considered range from 2 to 13 ; however, most of the data and images presented herein were obtained at $F r=7.3 \pm 0.2$, unless otherwise specified. After impact, once the ball was fully submerged, Reynolds number was used as a non-dimensional parameter to characterize cavity and sphere trajectory behaviour. Reynolds number was defined instantaneously along the sphere trajectory as $R e=V_{i} d / v$, where $V_{i}=V(t)$ is the instantaneous velocity of the ball in time $t$ and $v$ is the kinematic viscosity of the fluid.

The sphere's position in time was found using standard image processing crosscorrelation techniques on the whole sphere ( 74 pixel window size). A five-point Gaussian peak-fit was used to find the location of the maximum correlation peak, and thus determine the displacement of the sphere with subpixel accuracy. The peakfitting implementation was similar to that employed in Particle Imaging Velocimetry processing algorithms (e.g. Raffel et al. 1998). Using cross-correlation with peakfitting, the position of the sphere was determined accurate to within \pm 0.025 pixels. Conversion from pixels to metres yields an uncertainty of $\pm 1.93 \times 10^{-5} \mathrm{~m}(0.0193 \mathrm{~mm})$ in $x$ - and $y$-positions.

Measurement error for $x$ - and $y$-positions was affected predominately by image resolution and video acquisition rate: e.g. higher resolution, or more pixels per metre, would have given higher accuracy. The velocity and acceleration were determined by taking the first and second derivatives of a polynomial fit to the position data. The lowest-order curve fit to both $x$ - and $y$-positions was chosen such that higher order polynomials yielded nearly identical results for both velocity and acceleration, minimizing the error. This corresponded to a seventh-order polynomial fit with an $R^{2}$ value of 0.99 . Error estimates were between $2 \%$ and $4 \%$ in velocity, $5 \%-10 \%$ in acceleration and $5 \%-15 \%$ in lift and drag.

The rotation of the sphere was determined using an iterative, rotating crosscorrelation routine, which determined the mean and standard deviation of the angular position from the video sequences. Random non-uniform markings were drawn on the sphere with indelible marker to enhance the correlation algorithm. The correlation algorithm isolates one quadrant of the sphere in two sequential images and directly compares these isolated regions of interest, thus eliminating the need to shift the entire image. The quadrant from image 2 is rotated through a maximum rotation of $\pi / 4 \mathrm{rad}$, in increments of $\pi / 1800 \mathrm{rad}$. The incremental angular displacement between time steps corresponds to the angle where maximum correlation is found between image 1 and the rotated image 2 . The angular position data is found by summing the incremental angular displacement over time. Spin rate $\Omega(t) \mathrm{rad} \mathrm{s}^{-1}$ was found from the first derivative of a second-order polynomial fit to the angular position data. The mean spin rate and standard deviation were applied to a Student's $t$ distribution, which revealed error estimates of $4 \%-9 \%$ for $\Omega(t)$.

The spheres used in the bulk of this study were standard billiard balls made from phenolic resin with a mass ratio $m^{*}=m_{\text {sphere }} / m_{\text {fuid }}=1.74$. The surface roughness of the spheres was determined using model Tencor P-10 Surface Profilometer, sensitive to roughness of $\pm 0.01 \mu \mathrm{m}$. The size of the spheres was large compared to the profilometer's measurement range precluding measurement over the entire sphere surface, so only a fraction of the sphere could be tested at any given time. The theoretical 
smooth surface location is subtracted from the profilometer curves to determine r.m.s. values. The r.m.s. value for the phenolic resin spheres is $0.8 \pm 0.4 \mu \mathrm{m}$; the roughness to diameter ratio was $k / d=1.4 \times 10^{-5}$. The static contact angle made by a drop of fluid with the surface of the phenolic resin spheres was $90^{\circ} \pm 10^{\circ}$. The random markings on the spheres did not locally alter the average roughness or contact angle.

Additional materials and sizes of spheres were also tested to determine the effect of density and diameter on the splash and cavity physics. These spheres included smaller $(d=2.54 \mathrm{~cm})$ acrylic, ceramic and steel spheres. The mass ratios of the $2.54 \mathrm{~cm}$ spheres were $m^{*}=\{1.2,3.9,7.8\}$, for the acrylic, ceramic and steel spheres, respectively. The spheres were coated with a hydrophobic coating (Cytonics Corporation's WX2100 ${ }^{\mathrm{TM}}$ ) to have uniform surface properties; the r.m.s. surface roughness for all $2.54 \mathrm{~cm}$ spheres was $k=2.4 \mu \mathrm{m}$, and the static contact angle was $\theta_{c}=120^{\circ} \pm 10^{\circ}$. The data from these tests with the smaller projectiles are not the main focus of this paper, and therefore unless explicitly expressed, data and images presented in the following sections are for the standard billiard balls.

\section{Results and discussion}

\subsection{Overall cavity dynamics}

Following the discussions of May (1975), we consider the impact problem in five distinct stages: (1) the moment of contact, (2) the flow forming stage, (3) the open cavity and splash growth stage, (4) the closed cavity and pinch-off stage and (5) the collapsing cavity stage. While the details of each stage may vary with impact parameters, these five distinct stages persist for the case of the sphere impacting with spin.

Fundamentally, the initial stages of high-speed impact of any object on the free surface, with or without spin, are dominated by inertial effects. Figure 3 shows a sequence of images taken for a standard billiard ball spinning with $\Omega_{o}=199 \mathrm{rad} \mathrm{s}^{-1}$ $\left(S_{o}=1.1\right)$ and impacting the free surface with an initial velocity of $V_{o}=5.45 \mathrm{~m} \mathrm{~s}^{-1}$ $(F r=7.3)$. Each image in the sequence is separated by $\Delta t=10 \mathrm{~ms}$. The first image $(a)$ is taken $1 \mathrm{~ms}$ after impact. The sphere is already moving beyond stage 1 into stage 2 in figure $3(a)$.

An initial horizontal jet of fluid forms as the sphere impacts the free surface; this jet continues to extend radially outwards as the sphere descends into the fluid. The jet transitions from outwards to upwards growth during stage 2 when the sphere is submerged approximately one quarter of its diameter. A thin layer of fluid is driven around the lower surface of the sphere until it nears the equator, where it separates from the sphere to form the splash crown. Below a critical impact velocity and critical wetting angle, the flow remains attached until it surrounds the sphere completely and meets at the top, causing a jet of fluid to form upwards without the presence of an air cavity (Duez et al. 2007). For the spinning sphere, in the range of impact speeds considered for the standard billiard balls, the splash curtain appears to form symmetrically below $S \approx 0.30$, but above this value asymmetric growth of the splash curtain can be seen in the high-speed video sequences.

In stage 3 of the impact sequence (figure $3 c-f$ ) the entire ball passes below the ambient free surface and an open air cavity begins to form in its wake. The splash crown base, connected to the free surface, grows in diameter, with the top of the subsurface air cavity, and grows in height forming a vertical curtain. The subsurface air cavity both elongates vertically and grows radially outwards as the ball descends. At this stage the cavity is conical in shape, similar to the non-spinning case, but curved because of the spinning motion of the sphere which induces a lift force perpendicular to the ball trajectory. 


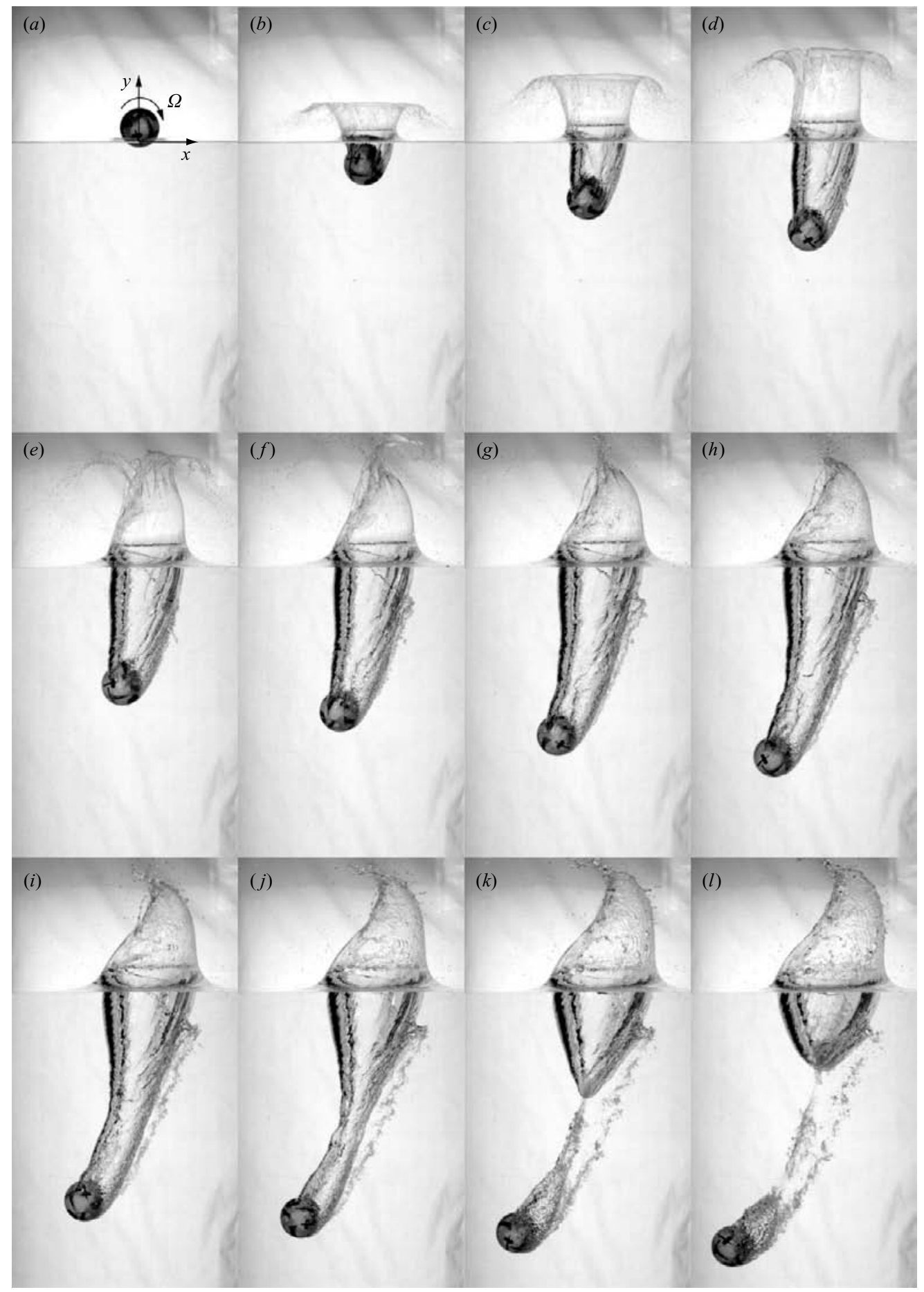

Figure 3. A sequence of images depicting the splash and air cavity formed in the wake of a spinning sphere impacting the water. The initial impact velocity is $V_{o}=5.4 \mathrm{~m} \mathrm{~s}^{-1}(F r=7.3)$ and the initial spin parameter is $S=1.1$. The first frame $(a)$ is $1 \mathrm{~ms}$ after impact; subsequent frames are $10 \mathrm{~ms}$ apart. (Digital video is available online for both side and top views of this run.) 
(a)

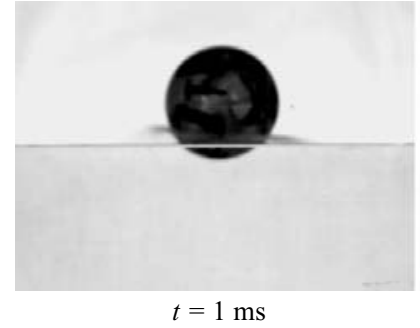

(b)

(c)

FIGURE 4. Splash asymmetry is already beginning to form due to spin $1 \mathrm{~ms}$ after impact $(a)$; the splash continues to grow in $(b)$ and $(c)$ leading to an asymmetric splash crown and dome. Sequence of images taken $\Delta t=2 \mathrm{~ms}$ apart, for a clockwise spinning sphere with initial impact parameters: $S=2.25$ and $F r=7.0$.

During stage 3, the sphere continues along its curved trajectory, bending the air cavity further. The subsurface air cavity is still open to the atmosphere and air flows in through the splash curtain resulting in a reduced pressure, which acts to draw the splash curtain inwards as it reaches its maximum height. The splash curtain continues to collapse inwards, eventually closing to form a dome (between figures $3 g$ and $3 h$ ).

In stage 4 the splash curtain is closed and no more air can flow into the cavity; however, the subsurface air cavity continues to stretch and curve under the movement of the spinning sphere. The cavity collapse for the case of the spinning sphere is similar to that of a non-spinning projectile as described in (Lee et al. 1997). Hydrostatic pressure of the surrounding fluid retards the outwards growth of the cavity and initiates the cavity collapse. The inwards inertia of the cavity forces it to neck down into a curved, yet bottle-like shape, reaching pinch-off (deep closure or deep seal) between figures $3(j)$ and $3(k)$. At pinch-off the cavity splits into two distinct closed cavities: a lower cavity still fully attached to the sphere, and an upper cavity connected to the free surface.

After pinch-off (stage 5) the cavity begins a rapid violent collapse (figure $3 j-k$ ). In both the lower and upper cavities, distinct jets of fluid eject away from the point of pinch-off in opposite directions similar to what is also seen by other researchers (e.g. Worthington \& Cole 1897; Lohse et al. 2004). The jet in the upper cavity bursts up through the free surface with significant velocity, pulling the upper cavity almost inside-out. The jet attached to the lower cavity is directed towards the sphere without an immediately obvious effect on the sphere's motion. The smaller lower cavity remains attached to the sphere (figure $3 l$ ). Ripples in the lower cavity are seen in the video sequences, comparable to those reported by Grumstrup, Keller \& Belmonte (2007). Eventually vortex shedding begins and the lower cavity starts to break up into bubbles that ascend to the surface.

\subsection{Effect of spin on cavity and splash asymmetry}

As spin rate increases from zero, the sphere follows an increasingly curved trajectory. The symmetry seen in the non-spinning cases gives way to asymmetric cavity and splash growth in the spinning cases. Asymmetry, due to spin, develops even for relatively low spin parameters and at early stages of splash formation. For example, the growth of the initial axial jet (at $t<1 \mathrm{~ms}$ ) is already asymmetric as it rises faster on the left side of the sphere, which is rotating out of the water, than the jet on the right side of the sphere. This is more evident in higher spin rate cases (figure 4). 
(a)

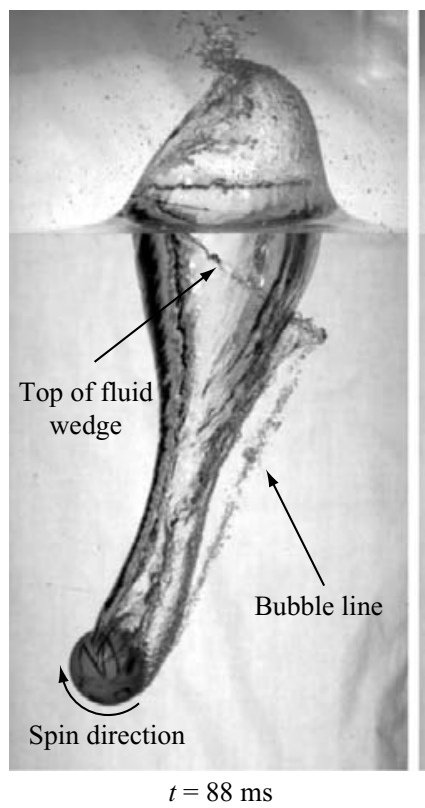

(b)

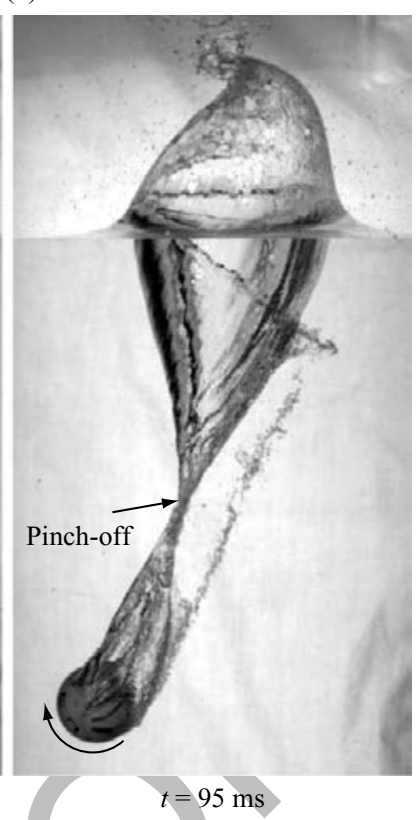

FIGURE 5. Two images taken at $t=88 \mathrm{~ms}(a)$ and $t=95 \mathrm{~ms}(b)$ after impact respectively, for initial impact parameters: $S=0.75$ and $F r=7.5$. In $(a)$ the top of the fluid wedge can be seen through the cavity as a grey line descending from the upper-left free surface to the lower right. A line of bubbles is ejected out of the right side of the cavity. Image $(b)$ is captured at the moment of pinch-off (deep closure); after pinch-off, two separate air cavities form.

As the splash curtain develops further, the left-to-right asymmetries persist (figure $3 a-d$ ) and are most evident when the curtain ceases to grow radially and starts its inwards collapse. At this point (figure $3 e-h$ ) the splash curtain appears to collapse earlier on the left side of the crown. The asymmetry of the splash dome is further exaggerated, as spin rate increases, and no outwards splash is formed on the left side of the cavity for spin parameters above a critical value of $S \approx 1.0$.

A line of bubbles can be seen ejecting out the right side of the cavity after surface closure and prior to deep closure (pinch-off). These bubbles persist for several frames and do not appear to have an effect on the overall cavity behaviour (figure 5). Upon close investigation it becomes clear that these bubbles are generated by a thin wedge of fluid, which has travelled from left to right inside of the cavity and has impacted the cavity wall, thus forcing air out along the line of impact. From the side view, the presence of a wedge is indicated by a dark diagonal line rooted near the free surface on the left side of the cavity and extending down to the right inside of the cavity; this line is the top of the fluid wedge (figure $5 a$ ).

The fluid wedge formation and bubble ejection are not persistent across all spin parameters. Figure 6 shows images taken at four distinct impact spin parameters, $S_{o}=\{0.3,0.75,1.1,1.4\}$. The top two image rows are synchronized in time and illustrate how the wedge formation varies from low to high spin parameters. Looking into the cavity from above, the top of the sphere is moving to the right and a small triangular wedge of fluid can be seen growing into the cavity. As the sphere spins it carries fluid, drawn from the cavity walls, along its equator into the cavity. The extruded fluid resembles a thin wedge, or sheet, that is anchored at the top on the 
Bird's-eye view $t=26 \mathrm{~ms}$

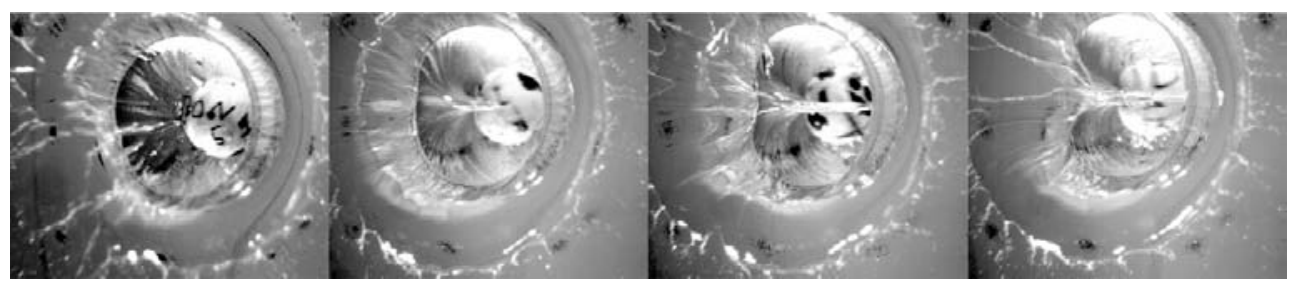

Close-up $t=26 \mathrm{~ms}$

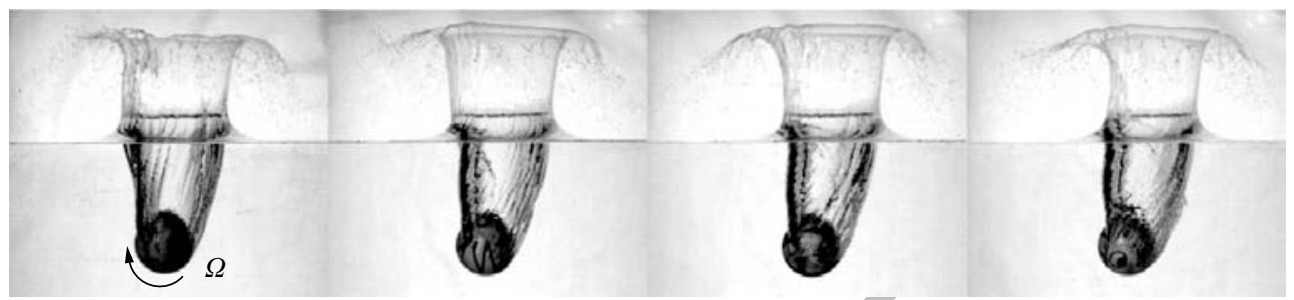

Side view $t=99 \mathrm{~ms}$

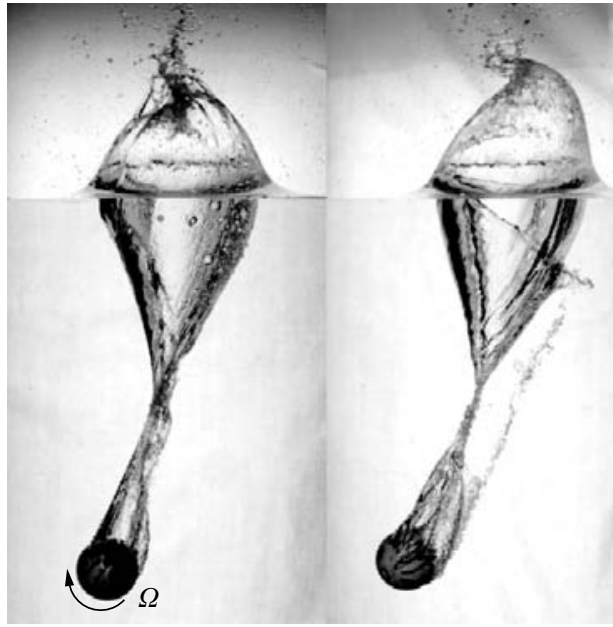

(a) $S_{o}=0.30$

(b) $S_{o}=0.75$

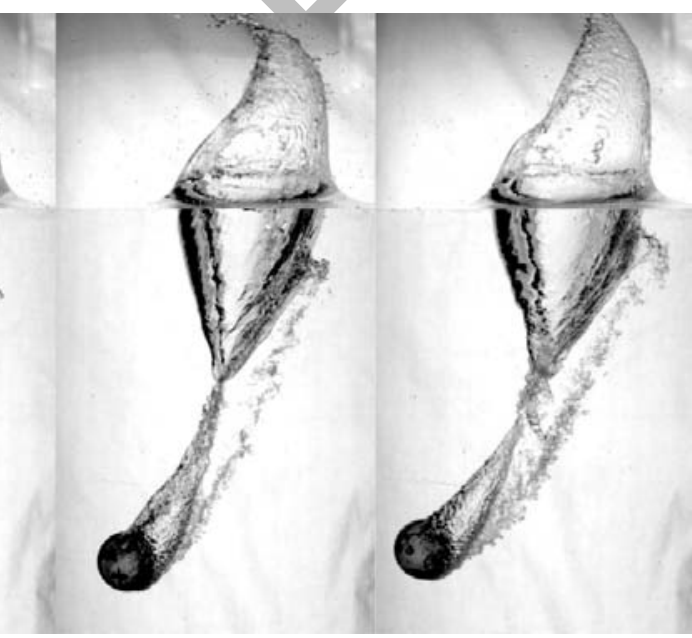

(c) $S_{o}=1.1$

FiguRE 6. Four water entry cases with increasing spin parameter (left to right). The 'bird's-eye' view (off-axis viewed from above) images in the top row are captured in synch with the images in the middle row; these images are taken at $t=26 \mathrm{~ms}$ after impact. The bottom row captures the cavity at $t=99 \mathrm{~ms}$ after impact, near the moment of pinch-off, showing the extent of the wedge formation for the four cases and pinch-off behaviour. Increased spin rate affects both the shape of the cavity cross-section and the overall splash symmetry. The spheres are spinning clockwise in the side views and the top of the sphere is moving left to right in the 'bird's-eye' view, drawing fluid across the cavity in the same direction. Froude number is $\mathrm{Fr}=7.3 \pm 0.2$ for all cases presented.

left side of the cavity and extends down the length of the cavity attached to the sphere surface at the lower end. For the lowest spin parameter presented $\left(S_{o}=0.30\right.$, figure $6 a$ ), the wedge formation is weak and does not fully extend across the cavity as it does in higher spin cases. In cases where spin parameters are less than $S_{o} \approx 0.30$, a distinct wedge of fluid is not formed; however, for all spin rates, images taken from 
(a)

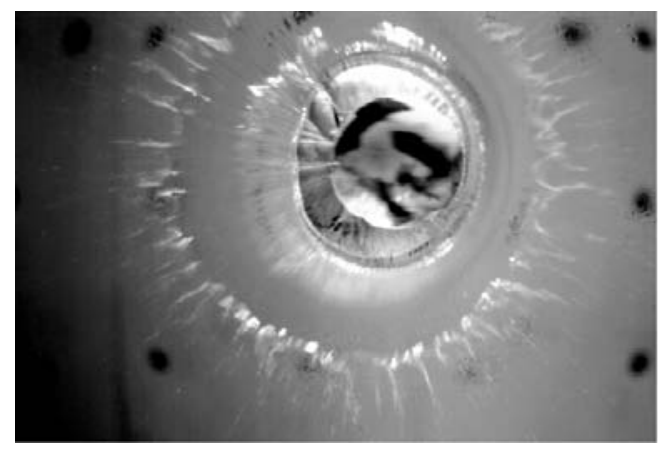

(c)

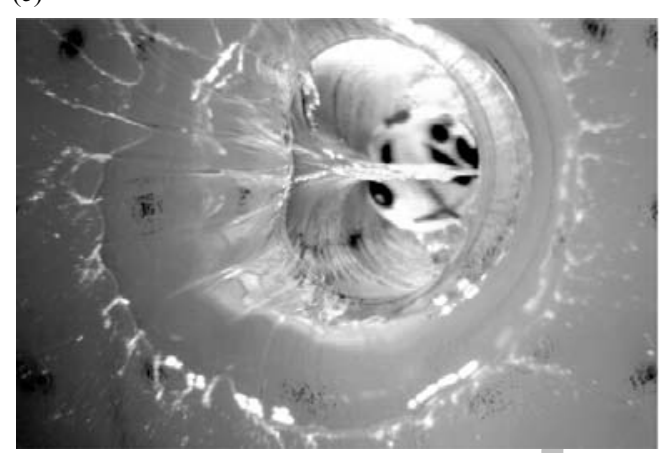

$t=26 \mathrm{~ms}$ (b)

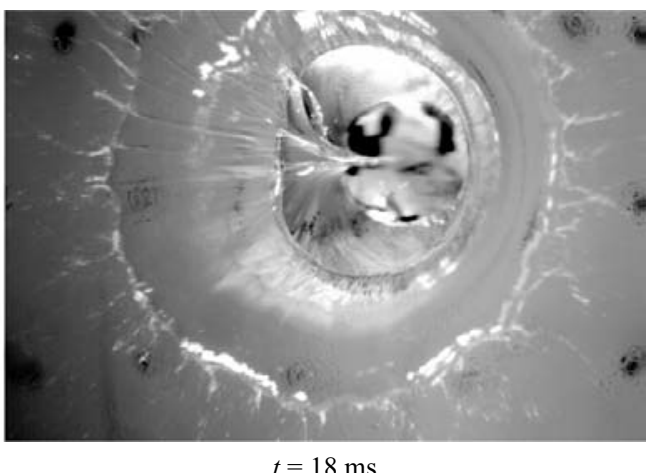

(d)

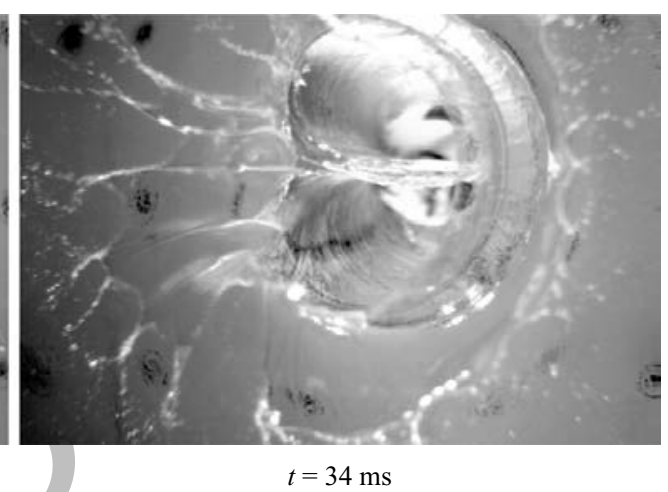

FIGURE 7. A 'birds-eye' view of a spinning sphere impacting the water surface at a downwards speed of $5.45 \mathrm{~m} \mathrm{~s}^{-1}(\mathrm{Fr}=7.3)$. The top of the sphere moves to the right with spin parameter $S_{o}=1.1$. Images are taken $8 \mathrm{~ms}$ apart. The rotation of the sphere draws fluid in from the left wall of the cavity $(a)$ towards the right, forming a wedge. The wedge increases in size as water continues to be brought in from the left $(18 \mathrm{~ms})(b)$, and thins along the equator of the sphere forming a sheet of water $(26 \mathrm{~ms})(c)$, which eventually impacts the right-hand side of the cavity $(34 \mathrm{~ms})$ in $(d)$.

the top reveal that the fluid is clearly drawn across the cavity in contact with the sphere, thus satisfying the no-slip condition.

At a later instant in time ( $t=99 \mathrm{~ms}$ after impact) the side views of the cavity reveal greater splash dome asymmetries with increasing spin parameter. The increase in spin causes the wedge to form earlier and travel at a faster velocity across the cavity. The progression of the fluid wedge from inception to the time at which the top of the wedge impacts the far cavity wall, is illustrated in figure 7 for the case $S=1.1$. The first image in this 'birds-eye' sequence shows vertical striations on the left wall of the cavity, which continue to grow into the cavity to form the wedge as the sphere descends. Even the early splash crown and air cavity opening, at $t=10 \mathrm{~ms}$, are slightly asymmetric. The shape of the cavity cross-section eventually evolves into a cardioid as the wedge extends across the cavity (figure $7 d$ ). At the higher spin rates the volume of fluid pulled from the left wall of the cavity increases, detracting from growth of the splash crown and affecting the cross-sectional shape of the cavity (figure 8). The splash crown growth and collapse are increasingly asymmetric at higher spin rates. For sufficiently high spin rates, no outwards splash occurs on the left side of the cavity; 
(a)

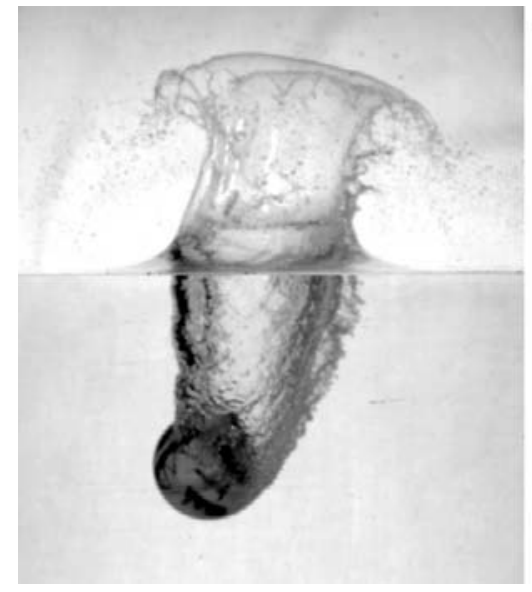

(b)

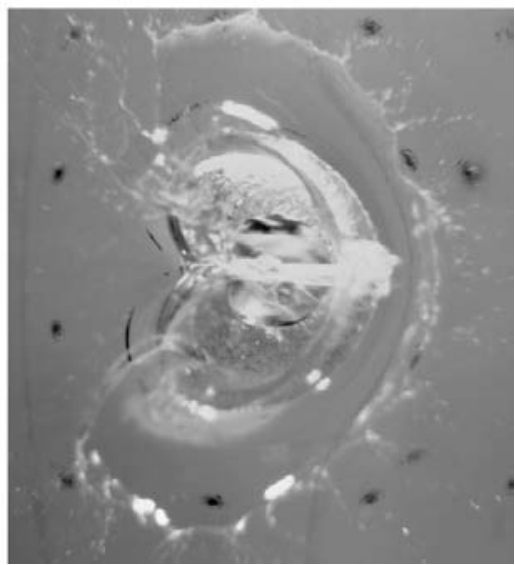

FiguRE 8. Side and top view for a high spin parameter case $S_{o}=2.25$, for $F r=7.0$, taken $26 \mathrm{~ms}$ after impact. The synched images show that the wedge has already crossed the cavity impacting the opposite cavity wall ejecting a line of bubbles on the right-hand side of $(a)$. (a) also shows the asymmetry of the splash curtain at this early stage of impact. $(b)$ from above shows a distinct cardioid shaped cavity and shows the wedge bisecting the cavity into two distinct halves. The spin parameter is sufficient enough to cause no outwards splash to occur on the left-hand side of the splash curtain as witnessed in $(b)$.

this behaviour is linked to the dynamic wetting angle and pinned contact line on the sphere as it both translates and rotates, and is the subject of ongoing investigation.

Just moments after impact, as the sphere continues to descend through the fluid, the spinning motion forces an already wetted section of the sphere to move upwards, dragging fluid along the surface of the sphere and into the cavity. Due to the rotational forces the fluid is drawn to the equatorial region. Assuming no-slip, the time the sphere takes to turn one-half of a full rotation, or $\pi$ radians, should be coincident with the time at which the wedge first impacts the cavity wall such that $t_{\pi}=\pi / \Omega_{o}$. During the time $t_{\pi}$, the sphere will have travelled some number of diameters $\alpha d$ below the free surface. Assuming that the sphere's forward velocity changes only minimally in this short time, then $\alpha d=V_{o} t_{\pi}$, such that

$$
\alpha=\frac{V_{o} \pi}{\Omega_{o} d}=\frac{\pi}{2 S_{o}} .
$$

If the sphere is located at some depth $y_{w}$ when the wedge impacts the wall then $\alpha=y_{w} / d$.

Plotting $y_{w} / d$ as a function of $V_{o} \pi / \Omega_{o} d$, reveals a linear relationship (figure $9 a$ ). However, the slope of the data is not equal to -1 as expected from (3.1). Instead the slope of the data is closer to -1.3 , indicating that the depth of the sphere at the moment of wedge impact, $y_{w}$ is over-estimated. The depth $y_{w}$ is determined by looking closely at the video sequences taken from the side view (camera \#2) and choosing the frame at which the bubbles first eject from the cavity near the sphere. There is a slight lag between the time that the leading edge of the wetted surface reaches the far wall and the time when a sufficient mass of fluid from the wedge hits the wall, causing air to eject from the cavity, which leads to an over estimation of $y_{w}$. For very low spin rates relative to impact velocity, it is possible that the wedge will 

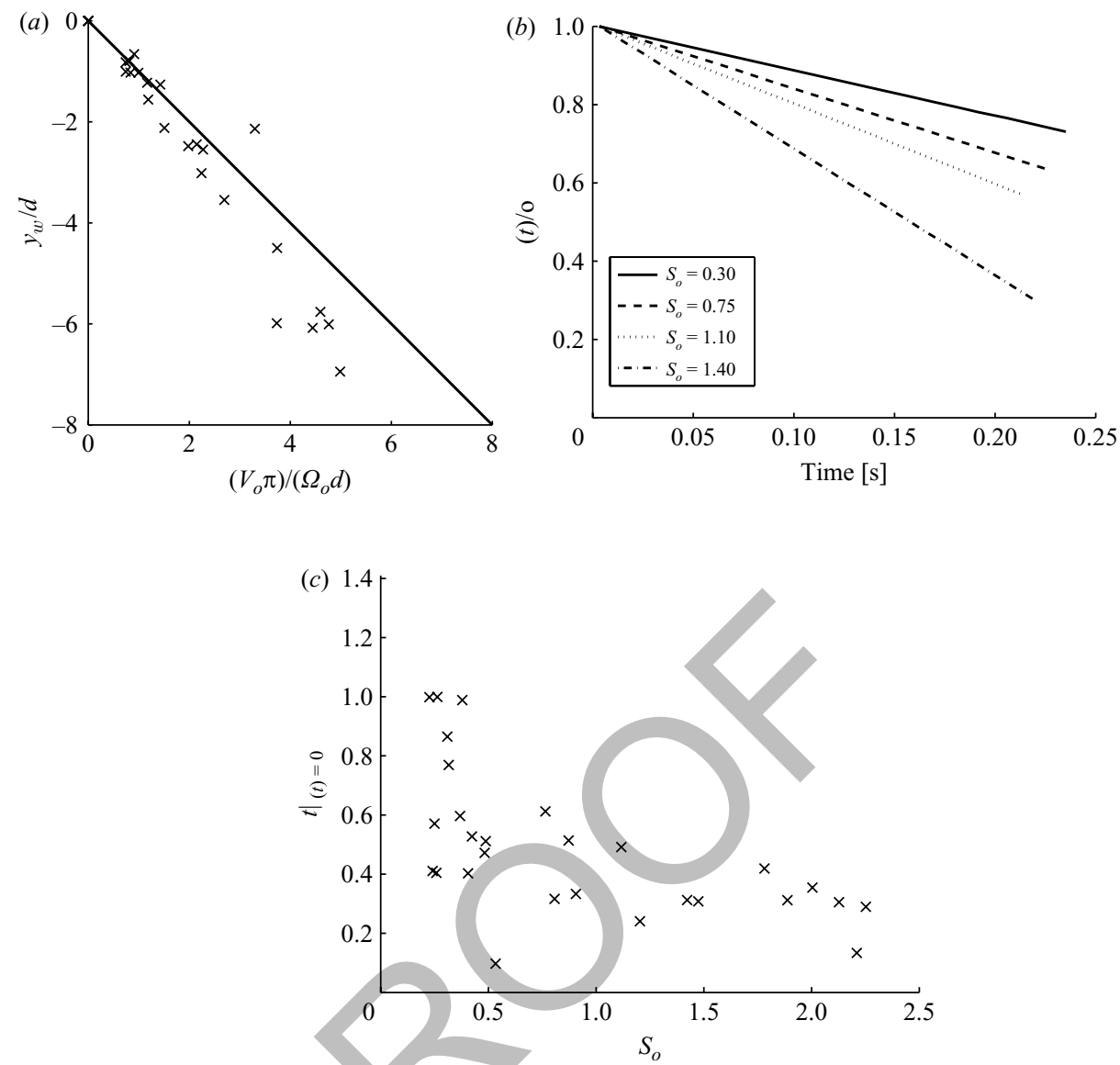

FIgURE 9. (a) The normalized depth of the sphere at which the wedge first impacts the opposite cavity wall as a function of the dimensionless parameter $V_{o} \pi / \Omega_{o} d$. Symbols are for experimental data, and solid line represents theoretical line with slope -1 , from (3.1). (b) The decay of spin rate, normalized by the impact spin rate, as a function of time for the four spinning cases from the standard billiard balls at $F r=7.3$. The highest spin rates see the largest reduction of spin in time. (c) The relaxation time, at which the spin rate would decay to zero, is found by extrapolating the data from $(b)$ down to the point at which the line crosses the horizontal axis.

not even reach the opposite side of the cavity before pinch-off, which is the case for data shown at $F r=7.3$ and $S \leqslant 0.3$.

Since the ratio of spin rate to downwards velocity strongly affects the wedge formation, the top of the fluid wedge (see figure $5 a$ ) forms a shallower angle to the free surface as spin rate increases. The leading, top corner of the wedge traverses across the cavity at approximately $60 \%$ of the tangential velocity $\Omega_{o} r$ of the sphere. As the cavity grows radially outwards and then begins collapsing, the distance the wedge must travel across the cavity grows and shrinks. It appears that the wedge is traversing the cavity at a nearly constant rate; however, the oscillation of the cavity wall makes the relative velocity between the wedge and opposing wall non-uniform. For cases where $S \gg 1$ the wedge impacts the side of the cavity early and violently, typically causing a line of bubbles, almost the length of the entire cavity, to eject from the cavity at nearly the same moment in time (figure 8). For lower spin parameters 
the initial wedge impact zone is typically near the top of the sphere, at the bottom of the cavity and progresses up the cavity wall towards the free surface.

As a result of the no-slip condition at the solid boundary, the fluid velocity at the sphere surface must match the sphere surface normal and tangential velocities, and thus fluid is dragged along the surface of the sphere as it rotates. Frictional viscous forces on the surface of the sphere act to reduce the spin rate of the spheres along the trajectory by causing a viscous torque that opposes rotation. Figure $9(b)$ plots the spin rate $\Omega(t)$ as a function of time, for four cases, normalized by the spin rate at impact. Ultimately, if allowed to travel in an infinite viscous fluid, the spheres would cease to spin due to the viscous torque opposing the rotation of the sphere. The rate of decay appears to have a dependency on spin parameter, increasing with increasing initial spin parameter, $S_{o}$.

Based on figure $9(b)$, it would be expected that the time that it would take a sphere to cease spinning, $\left.t\right|_{\Omega(t)=0}$, would decrease with increasing initial spin parameters. Figure $9(c)$ shows $\left.t\right|_{\Omega(t)=0}$, given the linear decay rates extracted from figure $9(b)$, as a function of initial spin parameter, $S_{o}$; this spin relaxation time is found by extrapolating the lines in figure $9(b)$ to the zero-crossing point on the time axis. Plotted as a function of impact spin parameter, the data reveal an asymptote beyond $S_{o} \approx 0.5$, where the relaxation time tends towards a value of $\left.t\right|_{\Omega(t)=o} \approx 0.3 \pm 0.1 \mathrm{~s}$.

The decrease in relaxation time, or increase in spin decay rate, as a function of increasing spin parameter is not wholly unexpected. As the spin parameter increases, so does the relative velocity on the surface of the sphere on the side where the tangential velocity due to rotation is additive with the sphere's forward motion. This acts to trip the boundary layer sooner on the side of the sphere with the highest relative velocity, thus transitioning it to a turbulent regime with higher viscous boundary layer drag. This increased viscous drag leads to a higher viscous torque that opposes the rotation and acts to slow the sphere at a faster rate than the spheres with a slower initial spin rate.

\subsection{Sphere trajectory}

The most obvious and anticipated change in behaviour of the spinning sphere, compared to the non-spinning case, is the curvature in its trajectory. The lift force induced by the rotating motion, coupled with forward velocity, moves the sphere along a curved path. Sphere trajectories for five different spin parameters, $S_{o}=\{0,0.3,0.75,1.1,1.4\}$, are plotted in figure 10 , for one impact velocity $\left(V_{0}=5.6 \pm 0.4 \mathrm{~m} \mathrm{~s}^{-1}\right)$; only position data after impact are presented. The $x$ - and $y$-positions are normalized by the diameter of the sphere $d$ and the free surface corresponds to a value of $y / d=0$.

The zero spin case shows a straight descent until after pinch-off. In figure 10, below $y / d \approx-7$, the sphere moves to the left; all non-spinning spheres tended to move away from their vertical trajectories at some time after pinch-off. This is most likely due to vortex-induced forces. Data in Govardhan \& Williamson (2005) indicate that tethered spheres, without spin, tend to present a sinusoidally oscillating motion in the axial direction due to vortices being shed in the wake. Before pinch-off, the cavity attached to the sphere retards classical vortex shedding from the sphere; thus it is only after pinch-off that the effects of vortex shedding would become noticeable.

The lift force significantly increases with spin in the range of spin rates investigated, and results in greater curvature of the sphere trajectory (figure 10). Path curvature is evident for all cases, indicating positive lifting force even at lower spin parameters (e.g. $S=0.30$ ). If spin parameter is held constant but Froude number increased, 


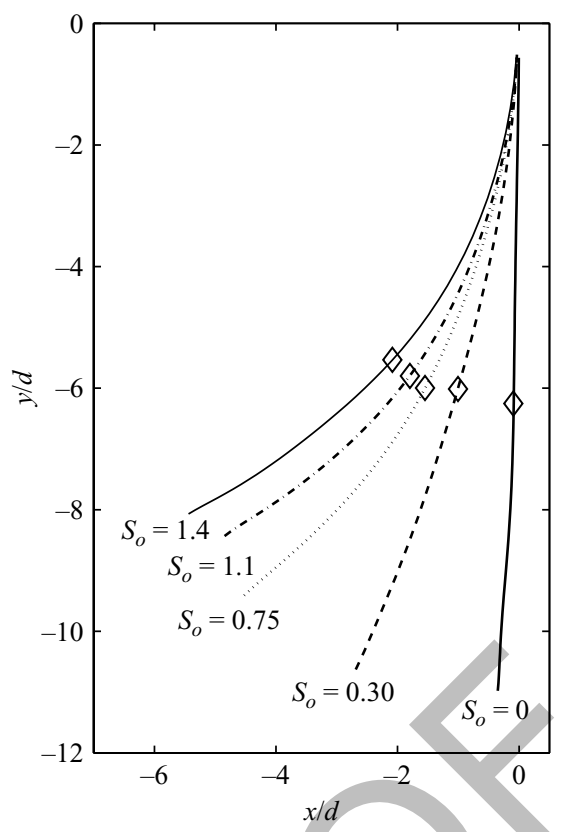

FIGURE 10. Five different trajectories of a billiard ball impact with the free surface. Each trajectory is marked by its corresponding spin parameter $\left(S_{o}=\Omega_{o} r / V_{o}\right)$ at impact. Froude number at impact was $F r=7.3 \pm 0.2$. The diamond marks the location of the sphere when pinch-off occurs for each case.

for a constant diameter sphere, the trajectories $x / d$ versus $y / d$ are very similar for each increasing Froude number (not shown). Differences in trajectories for increasing impact velocity are seen in the position as a function of time, as higher Froude number spheres reach deeper depths, and further horizontal excursions, earlier in time than cases with lower impact velocities. Since gravity plays a significant role in the motion of the sphere, the mass of the sphere should also be considered. Lower mass ratios should yield greater curvature assuming that the lift force results from increased circulation around the sphere. The mass ratio of the billiard ball used here is quite high, $m^{*}=1.74$, yet the curvature is still significant.

For the same impact parameters, $S_{o}$ and $F r$, lower mass ratios experience greater lateral excursion due to lift. The force of lift is expected to be similar for a given diameter, velocity and spin rate, but as the sphere travels along a curved trajectory the force of gravity tends to stretch the trajectory downwards and thwarts lateral movement; the gravitational force is greater for increasing body mass. Mass effects are considered using three different spheres, with constant diameter but varying mass ratios. Figure 11 shows the trajectories $x / d$ versus $y / d$, at $F r=6.7$ and $S_{o}=0.65$, for the three different types of spheres. The lighter spheres (acrylic) tend to have more curvature in their trajectories than the heavier spheres (ceramic and steel). For low mass ratios $m^{*}$ inertial forces are diminished compared to hydrodynamic forces, and added mass and lift forces become more significant. After the sphere leaves the camera's field of view, the acrylic sphere moves almost exclusively in a horizontal direction until its spin rate decreases significantly or it hits a tank wall, whereas the heaviest spheres always have some downwards motion. For $m^{*}$ near unity, e.g. acrylic spheres, lift force has a considerable effect on the sphere's trajectory and velocity 


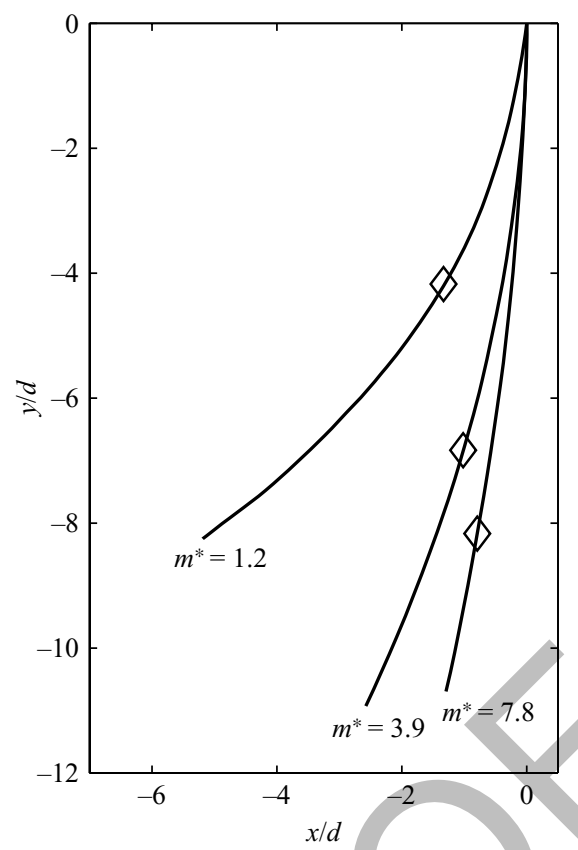

FIGURE 11. Trajectories for three $2.54 \mathrm{~cm}$ spheres with increasing mass ratios: acrylic $\left(m^{*}=1.2\right)$, ceramic $\left(m^{*}=3.9\right)$ and steel $\left(m^{*}=7.8\right)$. The three spheres impact the water at the same Froude number $(F r=6.7)$ and the same spin parameter $\left(S_{o}=0.65\right)$. The diamond markers indicate the depth of cavity pinch-off.

in the horizontal direction. For very high $m^{*}$, e.g. steel spheres, the inertial forces dominate and spin has little effect on the overall trajectory of the sphere.

Video images from the 'birds-eye' view and the side view for the acrylic, ceramic and steel spheres, captured at $t=21 \mathrm{~ms}$ after impact for $F r=6.7$ and $S_{o}=0.65$, are shown in figure 12. These images were obtained just prior to the time that the top two rows of images were acquired in figure 6 . The top-view images in figure $12(a-c)$ show a distinct difference in cavity cross-section for the three materials despite the identical impact parameters. No outwards splash is generated on the left side of the cavity for the steel spheres resulting in a distinct cardioid cross-sectional cavity shape, compared to the rounder cross-section of the acrylic and ceramic spheres. The spheres all have the same surface roughness, $k=2.4 \mu \mathrm{m}$, thus we should expect a similar frictional force to drag the fluid around as the sphere rotates. However, since wedge formation is dependent on the ratio of tangential velocity to downwards velocity (figure $9 a$ ), the qualitative difference in wedge formation makes sense. After impact, the acrylic spheres experience a more rapid deceleration compared to the ceramic and steel spheres since inertial effects are not as large, and thus have lower instantaneous velocities compared to the ceramic and steel spheres. The higher deceleration of the acrylic spheres is seen in the side-view images in figure $12(d-f)$; the steel sphere is deeper in the water than the acrylic sphere at the same instance after impact.

The heavier the sphere, the higher its kinetic energy is upon impact. The energy transferred to the fluid upon impact affects the splash and cavity formation. In the acrylic spheres a clean almost vertical splash forms and has not yet begun to collapse at $t=21 \mathrm{~ms}$ (figure $12 d$ ). The ceramic sphere splash crown is just beginning to collapse 
(a)

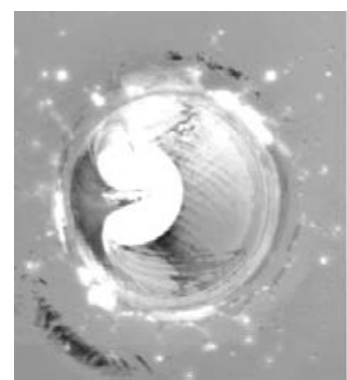

(d) (b)

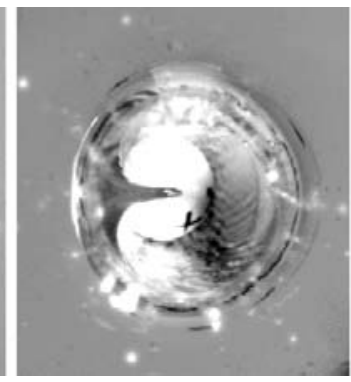

(e) (c)

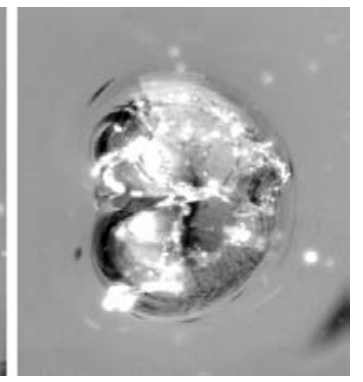

(f)
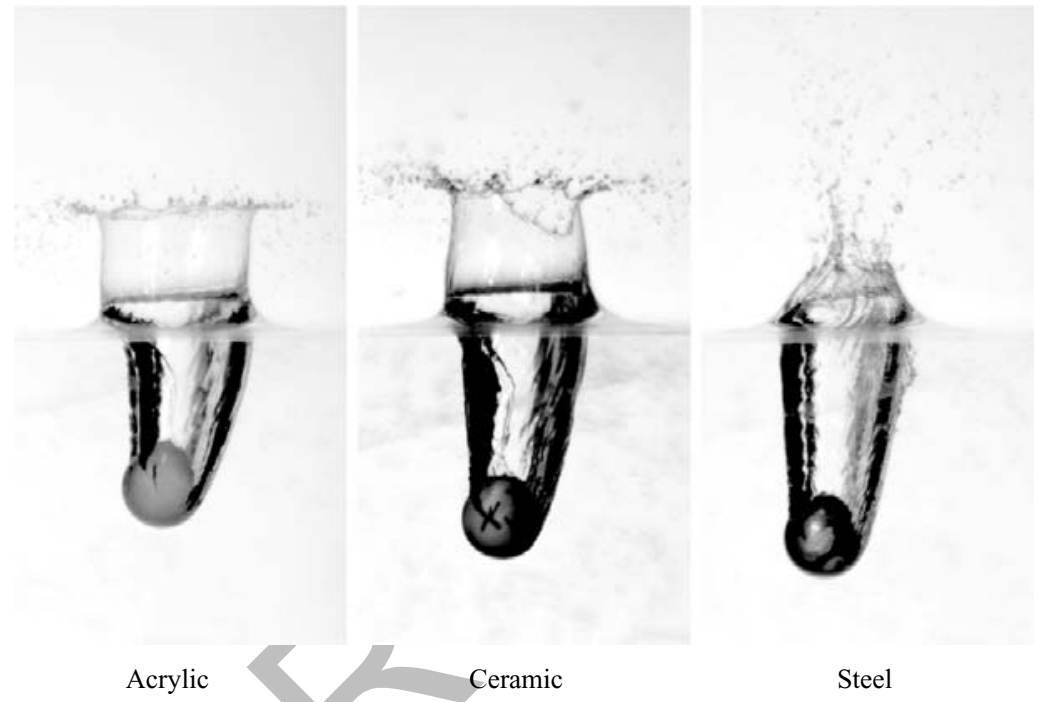

FIGURE 12. High-speed images of the cavity formation for three different $2.54 \mathrm{~cm}$ spheres with increasing mass ratio: acrylic $\left(m^{*}=1.2\right)$, ceramic $\left(m^{*}=3.9\right)$ and steel $\left(m^{*}=7.8\right)$. The spheres all impact the water at the same Froude number $(F r=6.7)$ and the same spin parameter $\left(S_{o}=0.65\right)$. Images of the 'birds-eye' view in the top row $(a-c)$ correspond to images of the side view in the bottom row $(d-f)$ and are captured at the same instance in time $(t=21 \mathrm{~ms})$. In images $(a-c)$ the equatorial line of the spheres is moving to the right and the spheres are rotating in a clockwise direction in images $(d-f)$.

(figure 12e) whereas for the steel sphere the splash crown has almost fully domed over (figure 12f). The bubble line, resulting from the wedge impacting the far wall of the cavity, can also be seen on the right side of the cavity only for the steel sphere in figure $12(f)$, but a faint wedge line can be seen through the cavity wall for both acrylic and ceramic spheres.

The diamond markers in figure 11 indicate that the depth of the sphere at the moment of pinch-off increases dramatically as mass ratio increases. The depth of pinch-off for the steel sphere is deeper than that of the ceramic and acrylic spheres. These trajectory plots do not reveal information about the velocity of the spheres' descent, but qualitative velocity differences are seen in the images in figure 12. As a result of the differences in velocities after impact, the time at which each sphere reaches a certain depth, e.g. pinch-off, changes dramatically between materials. Further discussion of this is found in the following section. 

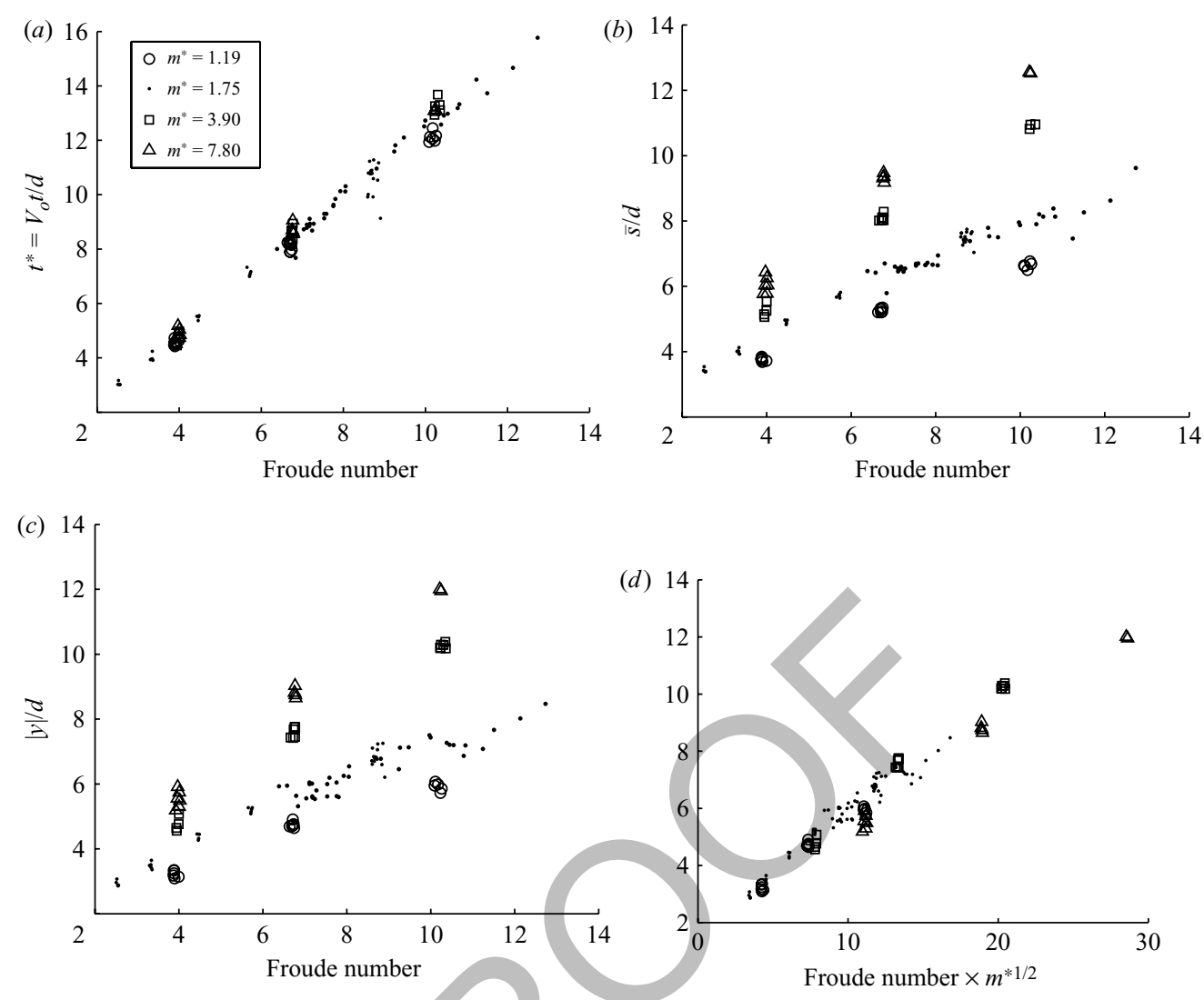

FIGURE 13. (a) Non-dimensional time to pinch-off plotted against Froude number and (b) normalized arclength-to-pinch-off as a function of Froude number; $(c)$ normalized depth to pinch-off as a function of Froude number and $(d)$ normalized depth to pinch-off as a function of Froude number and mass ratio. Data includes tests from standard billiard balls $(\cdot), 2.54 \mathrm{~cm}$ acrylic spheres $(\bigcirc), 2.54 \mathrm{~cm}$ ceramic spheres $(\square)$ and $2.54 \mathrm{~cm}$ steel spheres $(\triangle)$. Each material has a different mass ratio; $m^{*}$ is indicated in the legend for $(a)$.

\subsection{Cavity pinch-off}

Cavity deep seal is initiated by an imbalance in pressure inside and outside of the subsurface cavity. After surface closure the cavity continues to expand for some time as the sphere descends. Since air no longer flows into the cavity, the pressure inside must decrease if cavity expansion is considered to be an adiabatic isentropic process (Lee et al. 1997). Hydrostatic pressure outside the cavity grows with depth and the radial expansion of the cavity slows, and eventually stops, without new energy added to the system. The cavity starts to collapse and finally pinches off when it can no longer resist external pressures. The moment of pinch-off is taken when the subsurface air cavity has completely necked down; after pinch-off two distinct separated cavities form.

To find a scaling relationship for deep seal, the non-dimensional time to deep seal is considered. Figure 13(a) shows the relationship between non-dimensional time, $t^{*}=V_{o} t / d$ versus Froude number, and that data for all mass ratios, diameters and spin rates collapse onto one line. The slope of the linear fit to the data reveals that $t^{*}=5 / 4 \mathrm{Fr}$, over the range of mass ratios tested herein. However, in this figure 
the initial impact velocity dominates for both the $x$ - and $y$-axes, making $t^{*}$ a less than ideal scaling parameter. Gilbarg \& Anderson (1948) use a non-dimensional time scaling for deep seal using small spherical projectiles under varied atmospheric conditions, at Froude numbers much higher than those considered here. Glasheen $\&$ McMahon (1996) propose a single value of dimensionless time, $\tau=t \sqrt{g / r}$, for which deep seal reduces to a single number for all impact speeds and disk diameters. Data obtained herein reveals that $\tau=1.726 \pm 0.0688$ ( $\bar{x} \pm \sigma ; n=118$ trials), over the range of impact velocities and sphere diameters tested. This is similar to $\tau=1.74$ for spheres as reported by Gilbarg \& Anderson (1948), and can be contrasted with $\tau=2.285 \pm 0.0653$ ( $n=47$ trials) for disks as reported by Glasheen \& McMahon (1996).

The location of the sphere at the time of pinch-off is indicated in figure 10 by the diamond-shaped marker. The sphere's location at pinch-off is clearly affected by initial velocity and mass, but not spin. For constant Froude numbers, but increasing spin parameters, the depth $y / d$ of pinch-off increases only slightly, and the distance travelled by the sphere along its trajectory before pinch-off remains nearly constant. The distance travelled along the trajectory is the arclength-to-pinch-off distance, $\bar{s} / d=\sum d s / d$, where $d s$ is an elemental length along the trajectory path $s$.

For higher impact velocities and mass ratios, both the depth of pinch-off and arclength-to-pinch-off distances increase due to larger inertial effects and greater energy available to feed cavity growth. Both $\bar{s} / d$ and $y / d$ are plotted as functions of Froude number in figures $13(b)$ and $13(c)$. The data plotted in these figures also include, in addition to the standard billiard ball data, data obtained from three different 1 in. $(d=0.025 \mathrm{~m})$ spheres: acrylic $\left(m^{*}=1.2\right)$, ceramic $\left(m^{*}=3.9\right)$ and steel $\left(m^{*}=7.8\right)$. Data plotted includes all spin rate cases for each Froude number considered, including the non-spinning cases, revealing a minimal effect of spin on the deep seal phenomenon. While each specific mass ratio reveals a linear trend with Froude number, the data show that mass ratio is an important parameter affecting the depth and arclength at which pinch-off occurs. Taking into consideration the mass ratio effect, the normalized depth of pinch-off collapses neatly as a function of Froude number times the square root of the mass ratio (figure 13d).

\subsubsection{Force model}

3.5. Lift and drag forces on the spinning sphere

Lift and drag forces on the standard billiard balls are calculated using the position data acquired from the video sequences. The coefficients of lift $\left(C_{L}\right)$ and drag $\left(C_{D}\right)$ are found by normalizing the forces by $1 / 2 \rho V_{o}^{2} \pi r^{2}$. A force balance on the sphere is written in vector form based on the free body diagram in figure 14 $(a)$. The hydrodynamic forces $\left(\boldsymbol{F}_{H}\right)$ acting on the sphere are balanced by gravitational forces, added mass forces and surface tension:

$$
\boldsymbol{F}_{H}=m \boldsymbol{g}+\left(m+m_{a}\right) \boldsymbol{a}-\boldsymbol{F}_{B}-\boldsymbol{F}_{\sigma},
$$

where $m$ is the mass of the sphere, $\boldsymbol{g}$ is gravity, $m_{a}$ is the sphere added mass, $\boldsymbol{a}$ is the acceleration of the sphere and $\boldsymbol{F}_{B}$ is the buoyancy force. The force due to surface tension $\boldsymbol{F}_{\sigma}$ can be neglected as it is less than $1 \%$ of the gravitational force for the standard billiard balls. The buoyancy force $\boldsymbol{F}_{B}=\rho g \forall \hat{j}$, where $\forall$ is the sphere volume, and the added mass is found from $m_{a}=C_{m} \rho \forall$, where $C_{m}$ is the added mass coefficient. For this study $C_{m}$ was chosen to be constant over the entire run, $C_{m}=0.5$. The added mass coefficient $C_{m}$ likely changes over the course of the run, depending on how much of the sphere is submerged in water. Running the force model with 


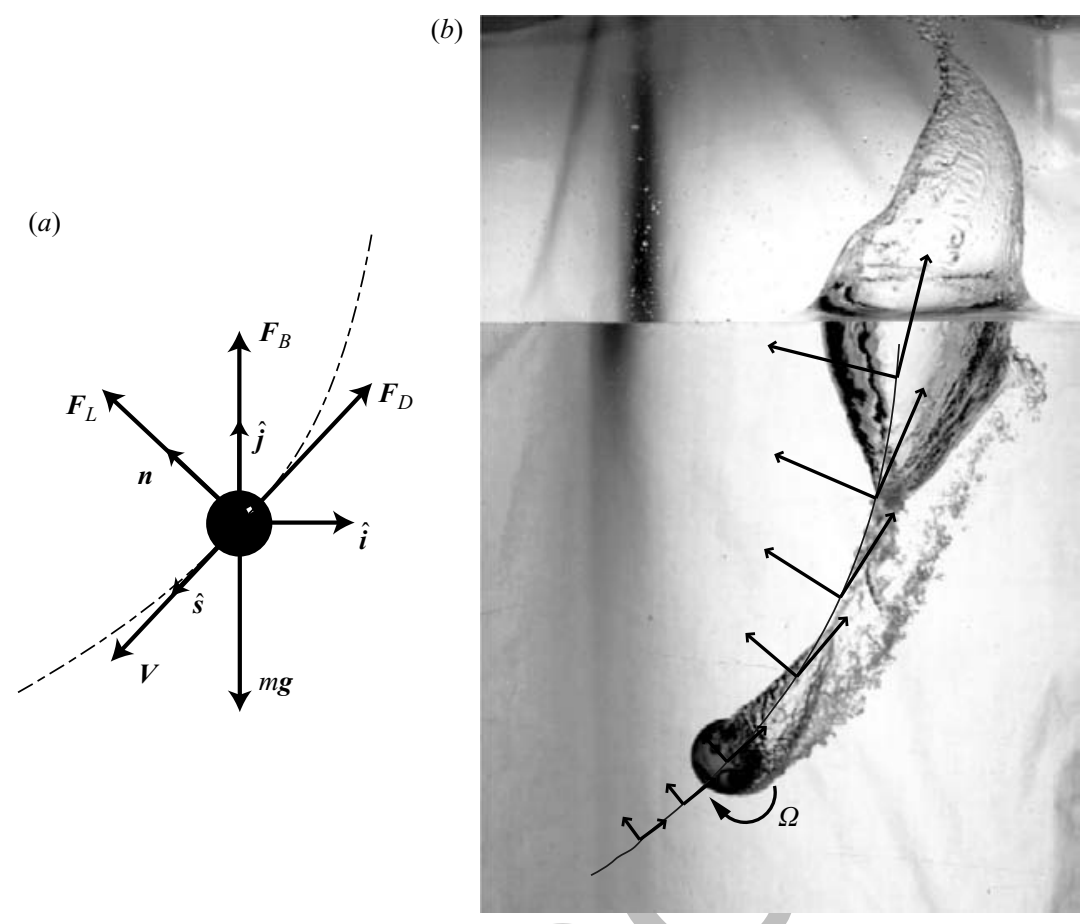

FIGURE 14. Free body diagram of the forces affecting the sphere after water entry is drawn in $(a)$. The dashed curve represents the sphere's trajectory. $(b)$ The changing magnitude of the lift and drag force components in time along the trajectory superimposed on one image taken from the same video sequence, $t=141 \mathrm{~ms}$ after impact. The vector origins correspond to the location of the center of the sphere along the trajectory at the same time step when the forces were calculated. Impact parameters for this case are $S_{o}=1.4$ and $F r=7.3$.

a constant drag coefficient $\left(C_{D}=0.4\right)$ for the non-spinning case shows that after the sphere is fully submerged, added mass coefficient could vary from as low as 0.2 , just after the cavity is formed, to as high as 0.5, after pinch-off. Choosing an added mass coefficient $C_{m}=0.25$, a $50 \%$ reduction in added mass, reduces the results for $C_{D}$ and $C_{L}$ by $8 \%-10 \%$, well within the error bounds of this study. For a mass ratio closer to unity $\left(m^{*} \approx 1\right)$ the added mass term will play a larger role and the choice of $C_{m}$ will be more critical. Force data is presented only for the standard billiard balls and assumes $C_{m}=0.5$.

To determine the lift and drag components of the forces, (3.2) can be broken into Cartesian vector components in the $x$ - and $y$-directions:

$$
F_{H} \hat{i}+F_{H} \hat{j}=m g \hat{j}+\left(m+m_{a}\right)(\ddot{y} \hat{j}+\ddot{x} \hat{i})-F_{B} \hat{j},
$$

where $\hat{i}$ and $\hat{j}$ are unit vectors in $x$ and $y$, respectively. Rewriting (3.3) in the reference frame of the sphere makes determining lift and drag forces along the curved trajectory more straightforward. The unit vector tangent to the sphere trajectory $\hat{s}$ is written in terms of the $x$ and $y$ components of instantaneous velocity $V_{i}$ :

$$
\hat{s}=\frac{\boldsymbol{V}_{i}}{\mid \boldsymbol{V}_{i}} \mid=\frac{V_{x}}{|\boldsymbol{V}|} \hat{i}+\frac{V_{y}}{|\boldsymbol{V}|} \hat{j} .
$$


The unit normal vector $\hat{n}$ is defined as the cross product of the unit tangent vector with the unit vector in the $z$-direction $\hat{k}$ :

$$
\hat{n}=\hat{s} \times \hat{k} .
$$

The forces of lift (transverse) $\boldsymbol{F}_{L}$ and drag (in-line) $\boldsymbol{F}_{D}$ can be determined from (3.2), in terms of unit vectors from (3.4) and (3.5) as

$$
\boldsymbol{F}_{L}=\left(\boldsymbol{F}_{H} \cdot \hat{n}\right) \hat{n},
$$

and

$$
\boldsymbol{F}_{D}=\left(\boldsymbol{F}_{H} \cdot \hat{s}\right) \hat{s},
$$

respectively. Lift is considered positive in the $+\hat{n}$ direction and causes curvature to the left in the images presented herein (the sphere is spinning in a clockwise direction).

The acceleration of the sphere is necessary to determine the force of lift $\left(\boldsymbol{F}_{L}\right)$ and drag $\left(\boldsymbol{F}_{D}\right)$. Directly differentiating the raw data does not result in accurate acceleration data and presents significant scatter and error. Thus a polynomial curve is fit to both the $x$ and $y$ data. A seventh-order polynomial fit was chosen for both the $x$ - and $y$-positions data; this was the lowest order to ensure convergence in acceleration for all cases. The $R^{2}$ values for the position fits are 0.99 . The acceleration in the $x$ - and $y$-directions is calculated from the second derivative of the polynomial fits to position. Similarly velocity is obtained by taking the first derivative of the polynomial fit. The $x$ - and $y$-positions, velocities and accelerations are plotted in figure 15 as a function of time for the five cases considered here.

Forces acting on the sphere change along the sphere's trajectory with changing velocity and acceleration. The coefficients of lift $\left(C_{L}\right)$ and drag $\left(C_{D}\right)$ are found by normalizing the forces by $1 / 2 \rho V_{o}^{2} \pi r^{2}$. Figure $14(b)$ shows a sphere at time $t=141 \mathrm{~ms}$ after impact $\left(S_{o}=1.4, \mathrm{Fr}=7.3\right)$. The sphere's trajectory is indicated by the curved line through the cavity. Superimposed on this line are pairs of orthogonal vectors representing the lift (normal) and drag (inline) forces; the length of the vector arrows indicate the relative magnitude of the forces on the sphere when it was located at the origin of the force vector pairs. As the sphere descends through the fluid column the forces of lift and drag decrease. Figure 14(b) also illustrates the asymmetry of the cavity formation around the sphere trajectory, with greater growth in the negative $\hat{n}$ direction than in the positive $\hat{n}$ direction.

\subsubsection{Forces as a function of Reynolds number}

Calculated values for lift and drag coefficients are plotted in figure 16 as function of instantaneous Reynolds number $\left(R=V_{i} d / v\right)$, for the five cases presented in figure 6 . Reynolds number is greatest at the moment of impact and varies with instantaneous velocity along the sphere's descent. The impact region is identified by the Roman numeral I. Surface closure is marked by a vertical grey band (region II) around Reynolds number of $R \approx 1.6 \times 10^{5}$. The range of Reynolds numbers, at which the five cases reach pinch-off and form two distinct cavities, is marked by the second darker vertical band (region III) around $R \approx 1.1 \times 10^{5}$.

For increasing spin rates, the overall lift coefficient increases with the circulation around the sphere. Looking at the two higher spin parameters, $S_{o}=1.1$ and 1.4, it appears that there may be a maximum possible amount of lift that can be gained by increasing spin; a plateau in lift coefficient is seen above $S \approx 1.5$ in the data for smooth spinning spheres reported by Maccoll (1928). Over the course of the sphere's decent the lift coefficients rise to a maximum at or near the point of pinch-off, in 

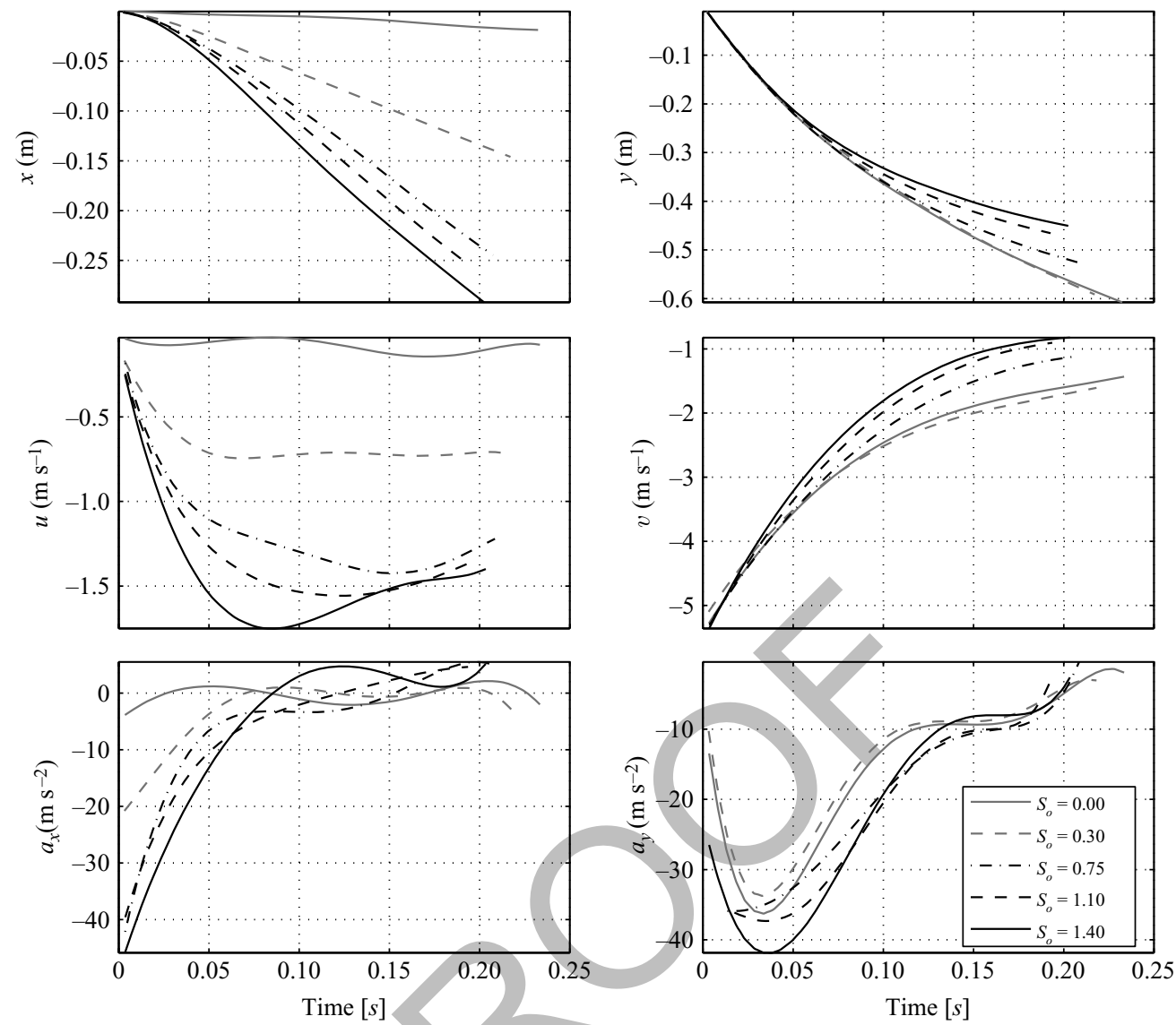

FIGURE 15. Position, velocity and acceleration in the $x$ - and $y$-directions as a function of time, resulting from a seventh-order polynomial fit to the raw position data for standard billiard balls with $F r=7.3$. Impact spin parameters are $S_{o}=\{0.0,0.3,0.75,1.1,1.4\}$; legend for $a_{y}$ is valid for all plots.

similar fashion to the drag coefficient. Drag coefficients, just after impact, are on par with the measured drag coefficient for a fully wetted sphere in flows at comparable Reynolds numbers (see figure 18). In the absence of vortex shedding, while the cavity is still fully intact, it might be expected that the drag coefficient would be lower than the fully submerged sphere at similar Reynolds numbers. Choosing a lower coefficient of added mass, e.g. $C_{m}=0.25$, reduces the overall drag coefficient to a value lower than published values for similarly rough, but fully submerged, spheres in the range of Reynolds numbers considered. The choice of added mass coefficient $C_{m}=0.5$ could account for the drag coefficient after impact being near to that of a fully submerged sphere.

\subsubsection{Forces as a function of spin parameter}

To further investigate the effect of spin on the forces incurred by the sphere, the coefficients of lift and drag are plotted as functions of instantaneous spin parameter $S(t)$ in figures 17 and 18, respectively. Figure 17 presents lift coefficient as a function of instantaneous spin parameter for five instantaneous Reynolds numbers, using data from figure 16(a). Data from smooth spheres measured by Maccoll (1928) and 

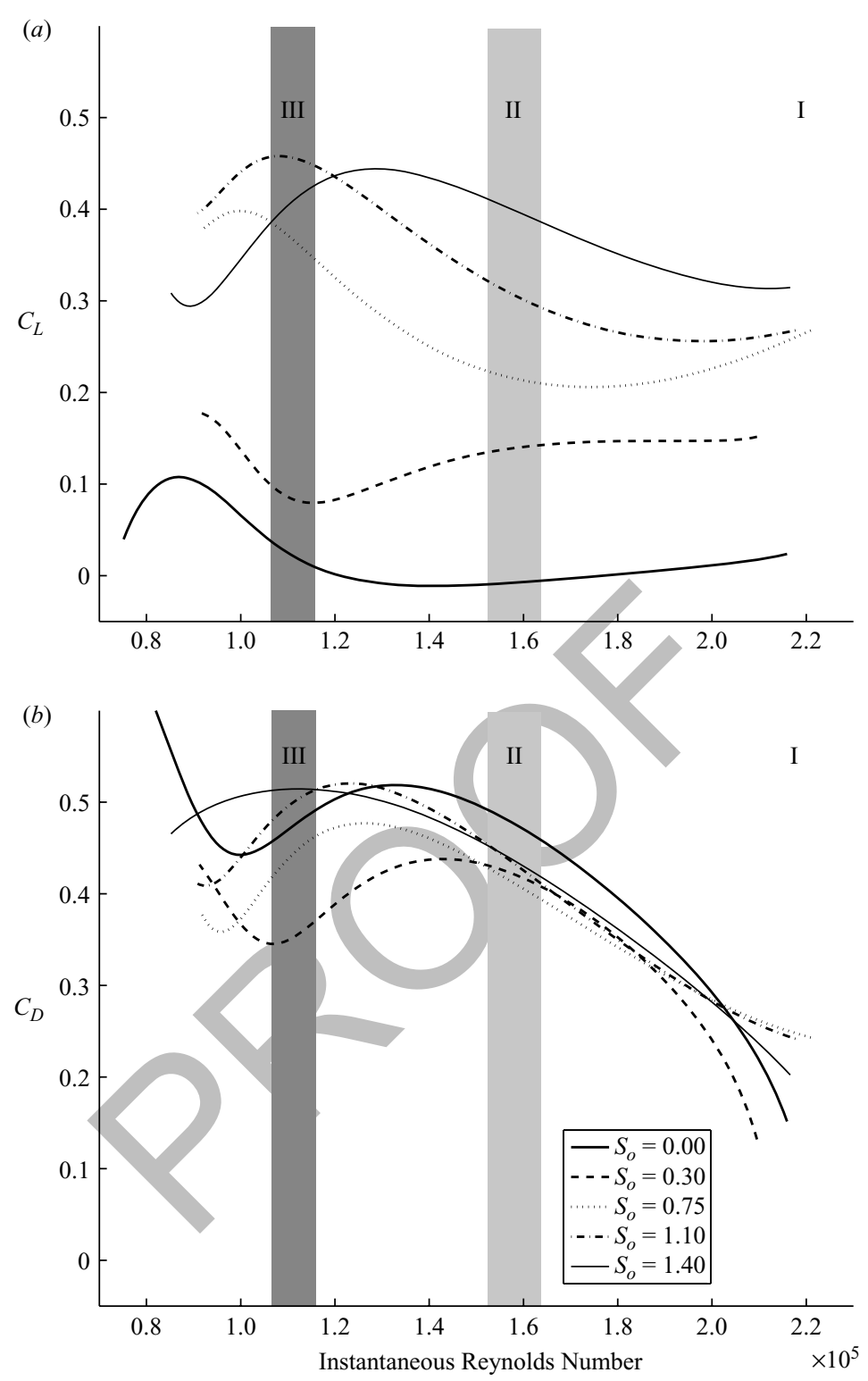

FIGURE 16. Lift $(a)$ and drag $(b)$ coefficients determined using the force model as a function of instantaneous Reynolds number $R=V(t) d / v$. Impact conditions for the five cases are: $S_{o}=\{0.0,0.3,0.75,1.1,1.4\}$ for $F r=7.3 \pm 0.2$. The legend in figure $(b)$ also corresponds with figure $(a)$.

dimpled spheres (golf balls) measured by Davies (1949) and Smits \& Smith (1994) are plotted for comparison.

Data show that the lift coefficient increases to a local maximum value of $C_{L} \approx 0.46$ at spin parameter $S(t)=1.8$ for Reynolds number $R(t) \approx 1.08 \times 10^{5}$; this instantaneous Reynolds number corresponds to the speed of the sphere near the time of pinchoff, but no obvious plateau has been reached. Data for higher instantaneous spin parameters were not obtainable for any given case, thus an overall maximum was 


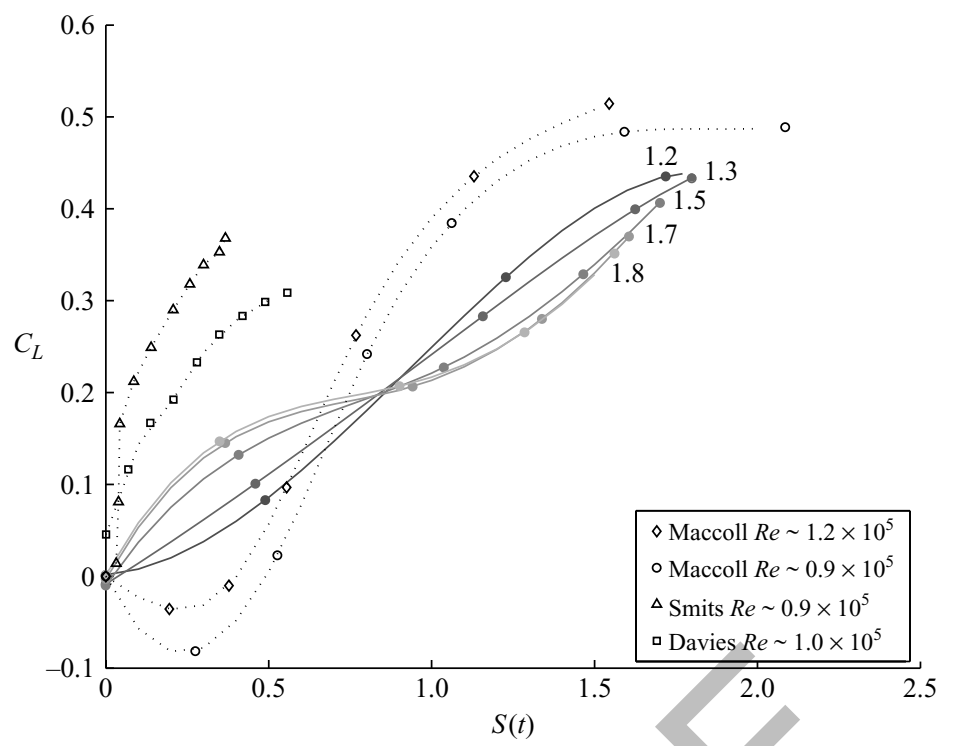

FIGURE 17. Coefficient of lift versus instantaneous spin parameter $S(t)$. Data for the spinning sphere impacting the free surface is plotted for five instantaneous Reynolds numbers between $R=1.2 \times 10^{5}$ and $1.8 \times 10^{5}$. These Reynolds numbers are taken from cases with different initial spin parameters but the same impact velocity, and correspond to the instantaneous and changing Reynolds number of the sphere along the trajectory. The number at the right of each curve is the corresponding Reynolds number divided by $10^{5}$. For comparison, data for dimpled spheres from Davies (1949) and Smits and Smith (1994) are plotted along with data from Maccoll (1928) for smooth spheres.

not obtained. The trend in lift coefficient is similar at higher Reynolds numbers, but the maximum coefficient is diminished with increasing Reynolds number. Higher Reynolds number curves in figure 17 correspond to points along the trajectory where the cavity is still intact and growing. The curvature of the trajectories increases as the sphere continues along its path, indicating that the lift forces due to spin could be more dominant, compared to cavity effects, as the sphere and cavity growth slows.

Lift coefficients obtained here follow similar trends, as a function of instantaneous spin parameter, to those found by Maccoll (1928); however, it is unclear whether this similarity continues above spin rate $S \approx 2.0$. In Maccoll (1928), negative lift coefficients were observed at very low spin parameters, below $S=0.35-0.45$. Negative lift was not witnessed in this study, nor in the golf ball studies done by other researchers, but was verified by Davies (1949) for very smooth spheres. Gilbarg \& Anderson (1948) noted that the average measured drag coefficients of the projectiles in their study were independent of cavity shape. Data presented here indicate that changes in cavity shape due to spin do effect drag; however, variable-added mass forces should also be considered in future studies.

Drag coefficients as a function of spin are plotted in figure 18, for the same five instantaneous Reynolds numbers used in figure 17. The value for drag coefficient in the non-spinning case $C_{D o}$ is calculated at each instantaneous Reynolds number using the force model from $\S 3.5 .1$. The $C_{D o}$ is subtracted from each curve in figure 18, such that each curve has zero drag at $S=0$. Thus, figure 18 closely represents the spin-induced drag forces on the sphere $C_{D S}$ without cavity effects. The total drag coefficient acting on the sphere is the sum of the zero-spin drag and the spin-induced 

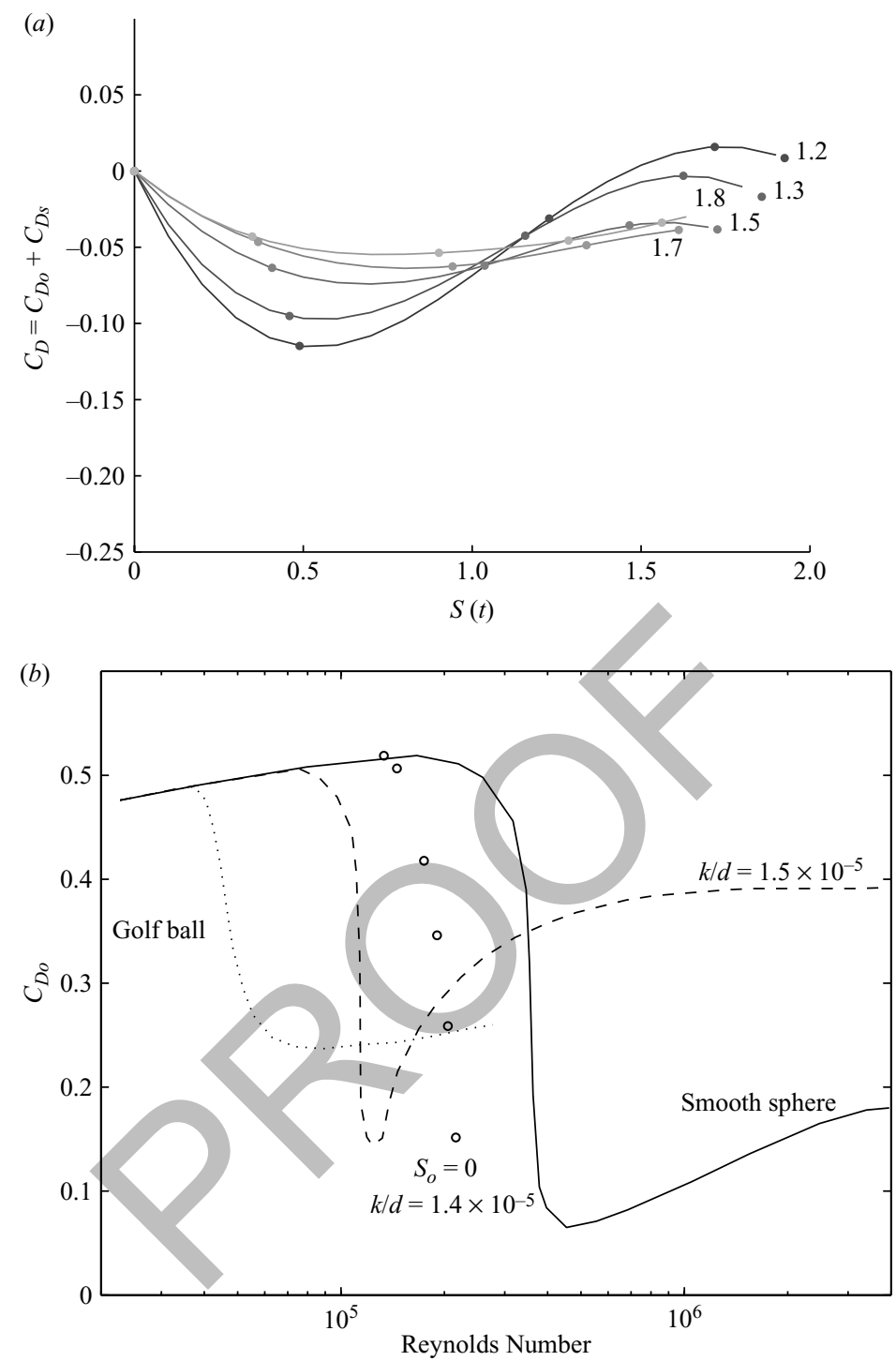

FIGURE 18. Coefficient of drag versus instananeous spin parameter at Reynolds numbers ranging from $1.2 \times 10^{5}-1.8 \times 10^{5}$. (a) shows the contribution due to spin only $\left(C_{D s}\right)$; the numbers to the right of the data curves are instantaneous Reynolds number divided by $10^{5}$. $(b)$ plots the drag coefficient $\left(C_{D o}\right)$ for the zero-spin water entry case with identical impact velocity as the spinning cases (circles). Standard drag coefficient data for smooth, roughened and dimpled spheres taken from Blevins (1984) is plotted for comparison.

drag: $C_{D}=C_{D o}+C_{D s}$. A negative $C_{D s}$ represents a reduction in drag coefficient due to spin. The effect of spin appears to decrease the drag coefficient over the course of a run compared to a non-spinning case, above an instantaneous Reynolds number $R \approx 1.2 \times 10^{5}$ and $S(t) \approx 1.5$.

Figure $18(b)$ presents the coefficient of drag calculated for the zero spin case $C_{D o}$ at $S_{o}=0$. Data are plotted along with standard drag curves for smooth and roughened spheres taken in flow tunnels taken from Blevins (1984). The cases investigated herein 
fall in the laminar-turbulent transition region, and figure 18(b) illustrates how the introduction of spin can easily tip the balance in favour of turbulent flow.

\section{Conclusions}

The effects of spin on the flight path of a sphere impacting into water are not ultimately surprising. However, high-speed video reveals the formation of unique and elegant splash and cavity morphologies when spin is introduced. As the spin rate of the sphere is increased, for a constant impact speed, the sphere's trajectory exhibits greater curvature, in a similar fashion to curveballs in sports like golf, cricket and baseball. The trajectories of higher mass projectiles are not as affected by spin as their lower mass counterparts. The bent cavity for the spinning case holds a similar, albeit curved, form compared to the non-spinning case, and the splash formation and collapse and the cavity pinch-off behaviours are fundamentally similar.

Unique to this problem, however, is the nature of the subsurface air cavity, and the formation of a secondary fluid feature, namely the fluid wedge, that forms in the cavity. Since there is no-slip between the sphere surface and the fluid, fluid is drawn along with the sphere, which is fully wetted after one-half a rotation, and the wedge is extruded into the cavity as the sphere descends. Data show that the ratio of spin rate to downwards velocity strongly affects the wedge formation. If allowed to travel in an infinite viscous fluid, the spheres would cease to spin due to the viscous torque opposing the rotation of the sphere. The rate of spin decay increases with increasing spin parameter, up to about $S_{o} \approx 0.5$, after which the spin relaxation time appears to plateau. The effect of mass ratio on wedge formation is played out in the instantaneous downwards speed of the sphere. Since the lighter spheres decelerate more rapidly than the heavier spheres, the instantaneous spin parameter for the light spheres is lower for the same impact velocity and the wedge formation is not as obvious.

Spin appears to have minimal effect on cavity pinch-off and collapse, compared to Froude number. The depth and arclength-to-pinch-off do not scale well with Froude number when mass effects are considered, yet scale well with Froude number times the square root of the mass ratio. Dimensionless time does collapse the pinch-off data as a linear function of Froude number, for all mass ratios; data for non-spinning cases collapse in an identical fashion to the data for spinning cases.

Several distinct regimes can be identified within the range of spin parameters studied. First, at zero spin rates the traditional water impact behaviours are identified. Using a force balance equation, the drag coefficient is found to increase for decreasing instantaneous Reynolds number, along the trajectory of the sphere, up to the point of pinch-off where the sphere separates from the large cavity. The cavity and splash formation and collapse are symmetric in the absence of spin and the calculated lift coefficients are zero.

For very low spin parameters $(0<S<0.35)$, where Maccoll (1928) notes negative lift coefficients, the spinning spheres studied here do not show negative lift, but instead tend to bend in the direction of positive lift, yielding the lowest calculated values for $C_{L}$. The drag coefficients calculated for the $S=0.30$ case were also the lowest of all the spin parameter cases run. Already at this low range of spin parameter, asymmetric splash and cavity formation and collapse are notable. No distinct wedge formation grows into the cavity, but the visible striations associated with wedge formation are evident (figure $6 a$; top row). Close observations of the 'birds-eye' videos indicate that there is no-slip between the fluid and the sphere at any spin rate. 
As spin parameter increases $(0.35<S<0.7)$, the lift data from Maccoll (1928) transitions to positive and the data recorded herein show a local minimum in drag coefficients for spin parameters between 0.5 and 0.6 , yet lift coefficient shows a steady increase in this region. The asymmetry in cavity and splash formation and collapse is exaggerated with increasing spin parameter, and a fully formed fluid wedge traverses across the cavity. Despite the wedge formation the cavity is still relatively round in cross-section at lower spin parameters, compared to spin parameters above $S_{o} \approx 0.7$. At the highest spin parameters $S_{o}>1.0$, the splash crown formation is significantly altered by spin. Minimal outwards splash arises from the left side of the impact region (as seen in the 'bird's-eye' images) and the cavity has a distinct and elegant cardioid shape and a dominant wedge that fully transects the cavity from surface all the way down to pinch-off.

Overall, the fundamental nature of water entry is not destroyed when spin is introduced, but instead altered in a unique fashion. Splash crown and subsurface air cavity do form and collapse in similar stages, but a new fluid wedge is formed that can dissect the cavity in half in the presence of spin. Preliminary tests show that static surface contact angles can affect the formation of the splash crown, as well as the fluid wedge; these effects warrant further investigation.

Funding for this work was provided through the ONR ULI (University Laboratory Initiative) grant number N00014-06-1-0445 by Theresa McMullen (ONR Code 333).

\section{REFERENCES}

Abelson, H. I. 1970 Pressure measurements in the water-entry cavity. J. Fluid Mech. 44, 129-144.

Alaways, L. W. \& Hubbard, M. 2001 Experimental determination of baseball spin and lift. J. Sports Sci. 19, 349-358.

Barkla, H. M. \& Auchterlonie, L. J. 1971 The Magnus or Robins effect on rotating spheres. J. Fluid Mech. 47, 437-447.

Bearman, P. W. \& Harvey, J. K. 1976 Golf ball aerodynamics. Aeronaut. Q. 27, 112-122.

BeLl, G. E. 1924 On the impact of a solid sphere with a fluid surface. Phil. Mag. 48, 753-764.

Bergmann, R., van der Meer, D., Stijnman, M., Sandtke, M., Prosperetti, A. \& Lohse, D. 2006 Giant bubble pinch-off. Phys. Rev. Lett. 96, 154505-4.

BIRKhoff, G. \& IsAaCs, R. 1951 Transient cavities in air-water entry. NAVORD Report No. 1490.

Blevins, R. D. 1984 Applied Fluid Dyanmics Handbook. Van Nostrand Reinhold Co.

Davies, J. M. 1949 The aerodynamics of golf balls. J. Appl. Phys. 20, 821-828.

Duez, C., Ybert, C., Clanet, C. \& Bocquet, L. 2007 Making a splash with water repellency. Nat. Phys. 3, 180-183.

FALTinsen, O. M. \& ZHAO, R. 1997 Water entry of ship sections and axisymmetric bodies. AGARD FDP and Ukraine Institute of Hydromechanics Workshop on High-Speed Body Motion in Water 24, 11 .

GAUdET, S. 1998 Numerical simulation of circular disks entering the free surface of a fluid. Phy. Fluids 10, 2489-2499.

Gilbarg, D. \& Anderson, R. A. 1948 Influence of atmospheric pressure on the phenomena accompanying the entry of spheres into water. J. Appl. Phys. 19, 127-139.

Glasheen, J. W. \& McMahon, T. A. 1996 Vertical water entry of disks at low Froude numbers. Phys. Fluids 8, 2078-2083.

Govardhan, R. \& Williamson, C. 2005 Vortex-induced vibrations of a sphere. J. Fluid Mech. 531, $11-47$.

Grumstrup, T., Keller, J. B. \& Belmonte, A. 2007 Cavity ripples observed during the impact of solid objects into liquids. Phys. Rev. Lett. 99, 114502.

vON KARMan, T. 1929 The impact on seaplane floats during landing. Technical Notes 321. National Advisory Committee for Aeronautics, Aerodynamic Institute of the Technical High School, Aachen. 
Kornhauser, M. 1964 Entry into water. In Structural Effects of Impact, ch. 2. Sartan Books.

Lee, M., Longoria, R. G. \& Wilson, D. E. 1997 Cavity dynamics in high-speed water entry. Phys. Fluids 9, 540.

Lohse, D., Bergmann, R., Mikkelsen, R., Zeilstra, C., van der Meer, D., Versluis, M., van der WeEle, K., van Der Hoef, M. \& Kuipers, H. 2004 Impact on soft sand: void collapse and jet formation. Phys. Rev. Lett. 93, 198003.

Maccoll, J. W. 1928 Aerodynamics of a spinning sphere. J. R. Aeronaut. Soc. 32 (213), 777-798.

MaY, A. 1951 Effect of surface condition of a sphere on its water-entry cavity. J. Appl. Phys. 22, $1219-1222$.

MaY, A. 1952 Vertical entry of missiles into water. J. Appl. Phys. 23, 1362-1372.

MaY, A. 1975 Water entry and the cavity-running behaviour of missiles. Final. Naval Surface Weapons Center White Oak Laboratory, Silver Springs, MD.

MaY, A. \& Hoover, W. R. 1963 A study of the water-entry cavity. Unclassified NOLTR 63-264. United States Naval Ordinance Laboratory, White Oak, MD.

May, A. \& Woodhull, J. C. 1948 Drag coefficients of steel spheres entering water vertically. J. Appl. Phys. 19, 1109-1121.

MaY, A. \& Woodhull, J. C. 1950 The virtual mass of a sphere entering water vertically. J. App. Phys. 21, 1285-1289.

MentA, R. D. 1985 Aerodynamics of sports balls. Annu. Rev. Fluid Mech. 17, 151-189.

Moghisi, M. \& SQuire, P. T. 1980 An absolute impulsive method for the calibration of force transducers. J. Phys. E: Sci. Instrum. 13, 1090-1092.

Moghisi, M. \& SQUiRe, P. T. 1981 An experimental investigation of the initial force of impact on a sphere striking a liquid surface. J. Fluid Mech. Digital Arch. 108, 133-146.

Newton, I. 1671 New theory about light and colors. Phil. Trans. R. Soc. 6, 3078.

Raffel, M., Willert, C., Willert, C. E. \& Kompenhans, S. 1998 Particle Image Velocimetry. Springer.

Richardson, E. G. 1948 The impact of a solid on a liquid surface. Proc. Phys. Soc. 4, 352-367.

Robbins, B. 1742 New Principles of Gunnery. Richmond (republished by Richmond in 1972, first printed by ed. Hutton).

Rosellini, L., Hersen, F., Clanet, C. \& BocQuet, L. 2005 Skipping stones. J. Fluid Mech. 543, 137-146.

Shi, H.-H., Iтон, M. \& TaKami, T. 2000 Optical observation of the supercavitation induced by high-speed water entry. J. Fluids Engng 122 (4), 806-810.

Smits, A. J. \& Smith, D. R. 1994 A New Aerodynamic Model of a Golf Ball in Flight. E. and F. N. Spon.

Thoroddsen, S. T. 2002 The ejecta sheet generated by the impact of a drop. J. Fluid Mech. 451, 373-381.

Thoroddsen, S. T., Etoh, T. G., TAKehara, K. \& Takano, Y. 2004 Impact jetting by a solid sphere. J. Fluid Mech. 499, 139-148.

Truscott, T. T. \& Techet, A. H. 2006 Cavity formation in the wake of a spinning sphere impacting the free surface. Phys. Fluids 18, 091113.

WATtS, R. G. \& FERRER, R. 1987 The lateral force on a spinning sphere: aerodynamics of a curveball. Am. J. Phys. 55, 40-44.

Worthington, A. M. \& Cole, R. S. 1897 Impact with a liquid surface, studied by the aid of instantaneous photography. Phil. Trans. R. Soc. Lond. Ser. A (Containing Papers of a Mathematical or Physical Character) 189, 137-148.

YARIN, A. L. 2006 Drop impact dynamics: splashing, spreading, receding, bouncing. Annu. Rev. Fluid Mech. 38, 159-192. 